\title{
11. MESOZOIC CALCAREOUS-NANNOPLANKTON STRATIGRAPHY OF THE CENTRAL NORTH PACIFIC (MID-PACIFIC MOUNTAINS AND HESS RISE), DEEP SEA DRILLING PROJECT LEG $62^{1}$
}

\author{
Pavel Čepek, Federal Institute for Geosciences and Natural Resources (Bundesanstalt für \\ Geowissenschaften und Rohstoffe), Hannover, Federal Republic of Germany
}

\section{INTRODUCTION}

Cretaceous sediments were recovered at all four sites (Sites 463-466) of the central North Pacific drilled during Leg 62 of the Deep Sea Drilling Project. One of the objectives was to get more information about the development of ocean plankton communities and early evolution of planktonic groups of the Mesozoic. In this article, the Cretaceous calcareous nannofossils from two areas of the central North Pacific (Mid-Pacific Mountains and Hess Rise) are listed and discussed. (The Cenozoic calcareous nannofossils are discussed by R. Schmidt in this volume.) Figure 1 shows the bathymetry and locations of Sites 463 to 466 . One site was drilled in the Mid-Pacific Mountains (Site 463) and three (Sites 464, 465 and 466) on Hess Rise. Coring was continuous at all sites. Mesozoic calcareous nannoplankton assemblages range on the Mid-pacific Mountains from Barremian to Early Maastrichtian, and on Hess Rise from Albian to Late Maastrichtian. (No calcareous nannofossils older than Barremian or Albian respectively were found.) The ages and the nannoplankton zone assignments of all drilled sites are shown in Figure 2. The stratigraphic correlations among Sites 464 to 466 (Hess Rise) are shown in Figure 3.

Determination of the calcareous nannoplankton was made with light microscopy on smear slides prepared from samples which were partly treated with an ultrasonic apparatus. Species recognized in the samples are listed in Table 1. Abundance and preservation of nannofossils were estimated for all samples studied. Species abundance was determined for all samples (except for Site 463) and recorded in Tables 2 (back pocket, this volume) to 6 . Calcareous nannoplankton species are listed in alphabetical order of species names on these tables. The ranges of index calcareous nannofossils of all sites are shown in Figures 4 to 7. On the same figures, a correlation is given on the zonation used with the zonation according to van Hinte (1976). The juxtaposition of the van Hinte (1976) zonation and the one used in this paper enables one to better correlate the coccolith stratigraphy with the biozonations of other fossil groups. The method utilized to express the abundance of coccolith specimens is a modification of the technique outlined in Hay (1970), Gartner (1972), and Čepek (1978). Smear slides were viewed at a magnifica-

\footnotetext{
${ }^{1}$ Initial Reports of the Deep Sea Drilling Project, Volume 62
}

tion of $\times 1560$. Samples with more than 1 specimen per field of view are listed as abundant (A); 1 specimen in 10 fields of view as common (C); 1 specimen in 50 fields of view as few (F); 1 specimen in 100 fields of view as rare (R); 1 specimen in 200 fields and more of view as very rare (VR).

The samples are listed in Tables 2 to 6 according to core, section, and interval (in $\mathrm{cm}$ ). For each sample, the depth has also been given in meters below sea floor. The abundance of nannoplankton assemblages was classified as abundant (A), common (C), few (F), and rare (R). The quality of preservation for the nannoplankton of each sample is recorded as good (G), moderate (M), or poor $(\mathrm{P})$.

\section{CALCAREOUS NANNOFOSSIL BIOSTRATIGRAPHY AND ZONATION}

The biostratigraphic nannofossil zonations of the Cretaceous used in this study are based upon van Hinte (1976), Martini (1976), and Thierstein (1976). The zonation according to van Hinte (1976) is based on the zonation of Bukry (1974), Roth (1973), and Thierstein (1973), and it is correlated with the zonations or biohorizons of radiolarians, benthonic foraminifers, planktonic foraminifers, calpionellids, and pelagic macrofossils.

Other calcareous nannofossils were used besides the marker species to determine the age of those sections in which the zones were impossible to recognize by calcareous-nannoplankton index species.

\section{Micula mura Zone}

Author: Martini (1969).

Definition: Interval from the first to the last occurrence of Micula mura (Martini).

Age: Late Maastrichtian.

Important common species: Micula mura (Martini), Lithraphidites quadratus Bramlette and Martini and Arkhangelskiella cymbiformis Vekshina.

Remarks: The guide fossil Micula mura (Martini) is common and well preserved. This zone is present only in Hole 465A. In Hole 465 this interval was missed.

\section{Lithraphidites quadratus Zone}

Authors: Čepek and Hay (1969).

Definition: Interval from the first occurrence of Lithraphidites quadratus Bramlette and Martini to the first occurrence of Micula mura (Martini).

Age: Middle to late Maastrichtian.

Important common species: Lithraphidites quadratus Bramlette and Martini and Arkhangelskiella cymbiformis Vekshina.

Remarks: This zone is present in both holes of Site 465 .

\section{Arkhangelskiella cymbiformis Zone}

Author: Perch-Nielsen (1972), emended Martini (1976).

Definition: Interval from the last occurrence of Tetralithus trifidus (Stradner) to the first occurrence of Lithraphidites quadratus Bramlette and Martini. 


\section{P. ČEPEK}

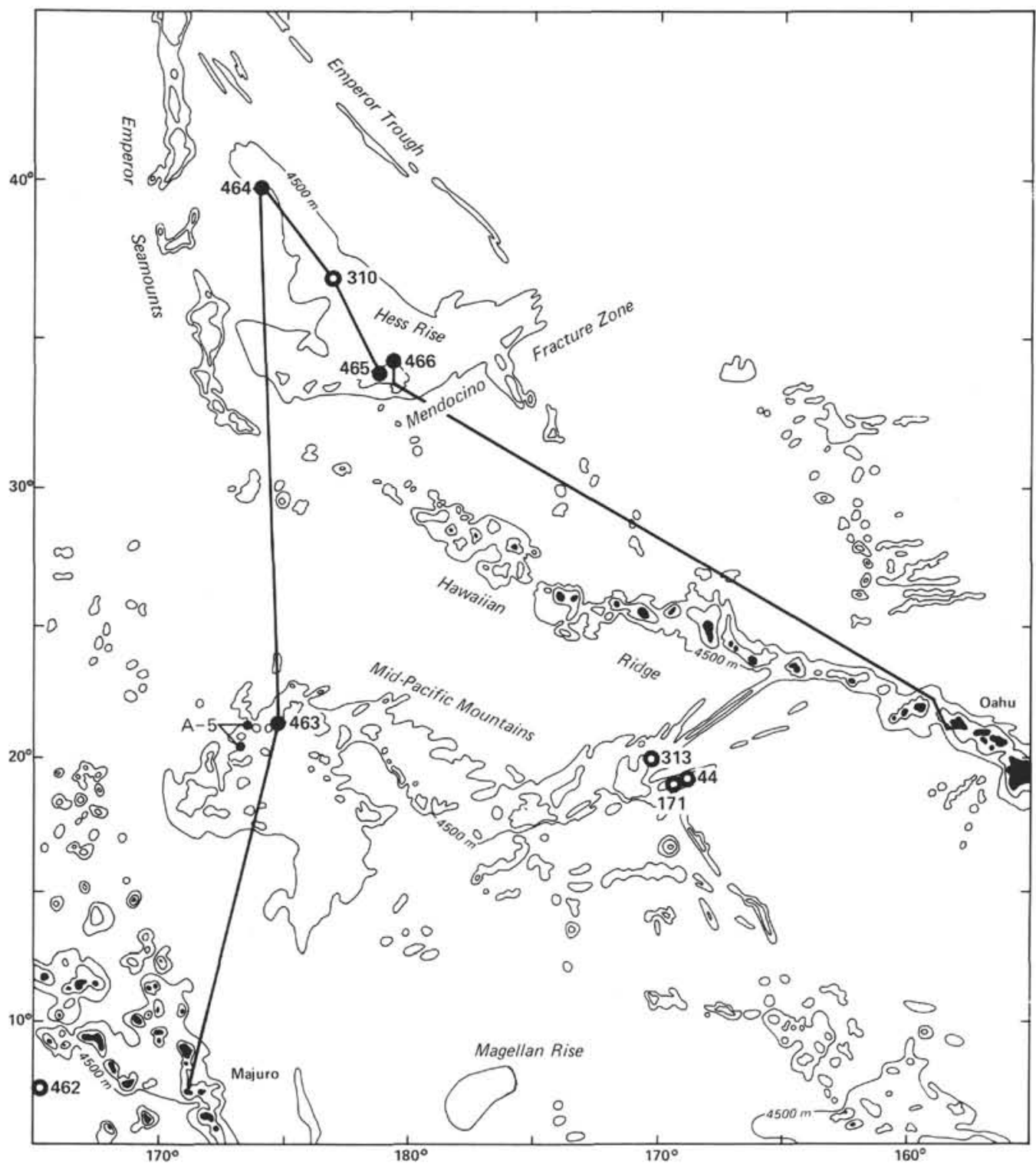

Figure 1. Index map of the central North Pacific, showing the drilled locations of Leg 62 and previous DSDP drill sites.

Age: Early Maastrichtian

Important common species: Arkhangelskiella cymbiformis Vekshina. Remarks: According to Martini (1976), the stratigraphic position of this zone is middle Maastrichtian. In this paper this zone is used for early Maastrichtian. This zone was identified in Holes 463 and $465 \mathrm{~A}$.

\section{Tetralithus trifidus Zone}

Authors: Bukry and Bramlette (1970).

Definition: Interval from the first to the last occurrence of Tetralithus trifidus (Stradner).

Age: Late Campanian to early Maastrichtian.

Important common species: Tetralithus trifidus (Stradner), Tetralithus gothicus Deflandre, Broinsonia parca (Stradner), and Eiffellithus eximius (Stover).

Remarks: The Tetralithus trifidus Zone was encountered in Holes 463, $465 \mathrm{~A}$, and 466 . In Holes 463 and $465 \mathrm{~A}$, the top of this zone is indicated by the last occurrence of Tetralithus trifidus (Stradner) and Tetralithus gothicus Deflandre. At Site 466, the top of this zone is missing. The last occurrence of Broinsonia parca (Stradner) in Hole $465 \mathrm{~A}$ is close to the top of this zone, which agrees well with the observations of Martini (1976) on Leg 33. In Hole 465A in the lower part of this zone, Eiffellithus eximius (Stover) is more common than in the upper part. Here this species is very rare and only present in one sample just below the top of this zone.

\section{Tetralithus gothicus Zone}

Author: Martini (1976).

Definition: Interval from the first occurrence of Tetralithus gothicus Deflandre to the first occurrence of Tetralithus trifidus (Stradner). Age: Late Campanian.

Important common species: Tetralithus gothicus Deflandre, Tetralithus aculeus (Stradner), Broinsonia parca (Stradner), Arkhangelskiella cymbiformis Vekshina, and Eiffellitkus eximius (Stover).

Remarks: The Tetralithus gothicus Zone was identified in Holes 463, 465A, 466. In all three holes, Tetralithus aculeus (Stradner) has its first occurrence at the same level as Tetralithus gothicus Deflandre. The former species seems to have the same importance for the delimitation of this zone in this area as Tetralithus gothicus Deflandre. This opinion does not conform to the zonation of Martini (1976). According to his results the first occurrence of Tetralithus aculeus (Stradner) is earlier than the first occurrence of 


\begin{tabular}{|c|c|c|c|c|c|c|c|c|c|c|c|}
\hline \multirow{3}{*}{ AGE } & \multirow{3}{*}{$\begin{array}{l}\text { CALCAREOUS-NANNOPLANKTON } \\
\text { ZONES }\end{array}$} & \multicolumn{10}{|c|}{ HOLE } \\
\hline & & \multicolumn{2}{|c|}{463} & \multicolumn{2}{|c|}{464} & \multicolumn{2}{|c|}{465} & \multicolumn{2}{|c|}{$465 \mathrm{~A}$} & \multicolumn{2}{|c|}{466} \\
\hline & & TOP & BASE & TOP & BASE & TOP & BASE & TOP & BASE & TOP & BASE \\
\hline \multirow{4}{*}{ Maastrichtian } & Micula mura & & & & & & & 3-3 & $3, \mathrm{CC}$ & & \\
\hline & Lithraphidites quadratus & & & & & $9, \mathrm{CC}$ & $11, \mathrm{CC}$ & $4, \mathrm{CC}$ & $4, \mathrm{CC}$ & & \\
\hline & Arkhangelskiella cymbiformis & $7 \cdot 3$ & $16, \mathrm{CC}$ & & & & & $6, \mathrm{CC}$ & $11-5$ & & \\
\hline & Tetralithus trifidus & $17-1$ & $22, \mathrm{CC}$ & & & & & $11, \mathrm{CC}$ & $19, \mathrm{CC}$ & $11-1$ & $11, \mathrm{CC}$ \\
\hline \multirow{2}{*}{ Campanian } & Tetralithus gothicus & 23-1 & $25-2$ & & & & & $20-1$ & $20, \mathrm{CC}$ & $12-1$ & $15, \mathrm{CC}$ \\
\hline & Broinsonia parca & & & & & & & $21 \cdot 3$ & 21.4 & $16 \cdot 1$ & $16-3$ \\
\hline & \multirow{4}{*}{ (unzoned) } & \multirow[t]{3}{*}{$25, \mathrm{CC}$} & \multirow[b]{3}{*}{$30, \mathrm{CC}$} & & & & & & & \multirow{4}{*}{$16, \mathrm{CC}$} & \multirow[b]{4}{*}{$23, \mathrm{CC}$} \\
\hline Santonian & & & & & & & & $21, \mathrm{CC}$ & $23, \mathrm{CC}$ & & \\
\hline Coniacian & & & & & & & & & & & \\
\hline Turonian & & $31, \mathrm{CC}$ & $37, \mathrm{CC}$ & & & & & & & & \\
\hline Cenomanian & Lithraphidites alatus & $38-1$ & $50-1$ & $11-1$ & $18, \mathrm{CC}$ & & & $26-1$ & 27, CC & & \\
\hline \multirow{3}{*}{ Albian } & Eiffellithus turriseiffeli & $50, \mathrm{CC}$ & $52, \mathrm{CC}$ & $18, \mathrm{CC}$ & $24-1$ & & & $28-1$ & $40-1$ & $29-2$ & $35, \mathrm{CC}$ \\
\hline & Prediscosphaera cretacea & $53-1$ & $55-1$ & $25, \mathrm{CC}$ & $26-1$ & & & & & & \\
\hline & Parhabdolithus angustus & $55-1$ & $65 \cdot 2$ & $27 \cdot 1$ & $27 \cdot 1$ & & & & & & \\
\hline Aptian & Chiastozygus litterarius & $65, \mathrm{CC}$ & $78-1$ & & & & & & & & \\
\hline Barremian & Micrantholithus obtusus & $79-1$ & $92, \mathrm{CC}$ & & & & & & & & \\
\hline
\end{tabular}

Figure 2. Age determinations and zone assignments for the Cretaceous calcareous nannoplankton of Sites 463 to 466 .

Tetralithus gothicus Deflandre. Later studies and correlations in this area will show whether this occurrence together is the result of a small hiatus corresponding to the Tetralithus aculeus Zone, in accordance with the zonation of Martini (1976).

\section{Broinsonia parca Zone}

Author: Verbeek (1976).

Definition: Interval from the first occurrence of Broinsonia parca (Stradner) to the first occurrence of Tetralithus aculeus (Stradner). Age: Early to middle Campanian.

Important common species: Broinsonia parca (Stradner), Arkhangelskiella cymbiformis Vekshina and Parhabdolithus regularis (Gorka).

Remarks: This zone was identified only in Holes 465A and 466. The top of this zone is marked by the first occurrence of Tetralithus aculeus (Stradner), which in the central North Pacific corresponds to the level with the first occurrence of Tetralithus gothicus Deflandre. In both holes, the first occurrence of Broinsonia parca coincides with the first occurrence of Parhabdolithus regularis.

\section{TURONIAN TO SANTONIAN}

The abundant but predominantly poorly preserved assemblages of the Turonian to Santonian are characterized by the lack of index species which are indispensible for zonation. It is impossible to use a detailed zonation such as that published by van Hinte (1976) in this interval. The upper limit of this zonal interval is determined by the first occurrence of Broinsonia parca (Stradner) [in Hole 463 by the first occurrence of Tetralithus gothicus (Deflandre)], and the lower limit by the last occurrence of Lithraphidites alatus Thierstein.

\section{Lithraphidites alatus Zone}

Author: Roth (1973), modified in this paper.

Definition: Interval from the first occurrence of Lithraphidites alatus

Thierstein to the last occurrence of Lithraphidites acutum Verbeek and Manivit.

Age: Uppermost Albian to Cenomanian (lowermost Turonian).
Important common species: Lithraphidites alatus Thierstein, Lithraphidites acutum Verbeek and Manivit, and Parhabdolithus asper (Stradner).

Remarks: The Lithraphidites alatus Zone was encountered in Holes 463, 464 and 465A. Verbeek (1977) defined the Lithraphidites acutum Zone as the interval from the first occurrence of Lithraphidites acutum Verbeek and Manivit to the first occurrence of Gartnerago obliquum (Stradner). The range of the Lithraphidites acutum Zone is middle to late Cenomanian, which means that this zone could be used as a subzone of the Lithraphidites alatus Zone. The first and last occurrences of Lithraphidites alatus Thierstein in Holes 463 and 465A lie deeper than the first and last occurrences of Lithraphidites acutum Verbeek and Manivit. In Hole 464, Lithraphidites alatus Thierstein is missing.

\section{Eiffellithus turriseiffeli Zone}

Author: Thierstein (1971).

Definition: Interval from the first occurrence of Eiffellithus turriseiffeli (Deflandre and Fert) to the first occurrence of Lithraphidites alatus Thierstein.

Age: Late Albian

Important common species: Eiffellithus turriseiffeli (Deflandre and Fert), Podorhabdus albianus Black, Prediscosphaera cretacea (Arkhangelsky), and Parhabdolithus asper (Stradner).

Remarks: The Eiffellithus turriseiffeli Zone is the only Cretaceous zone present in all Sites (463-466) drilled during Leg 62.

\section{Prediscosphaera cretacea Zone}

Author: Thierstein (1971).

Definition: Interval from the first occurrence of Prediscosphaera cretacea (Arkhangelsky) to the first occurrence of Eiffellithus turriseiffeli (Deflandre and Fert).

Age: Middle Albian

Important common species: Prediscosphaera cretacea (Arkhangelsky), Parhabdolithus angustus (Stradner), and Podorhabdus albianus Black.

Remarks: This zone was identified in Holes 463 and 464. 


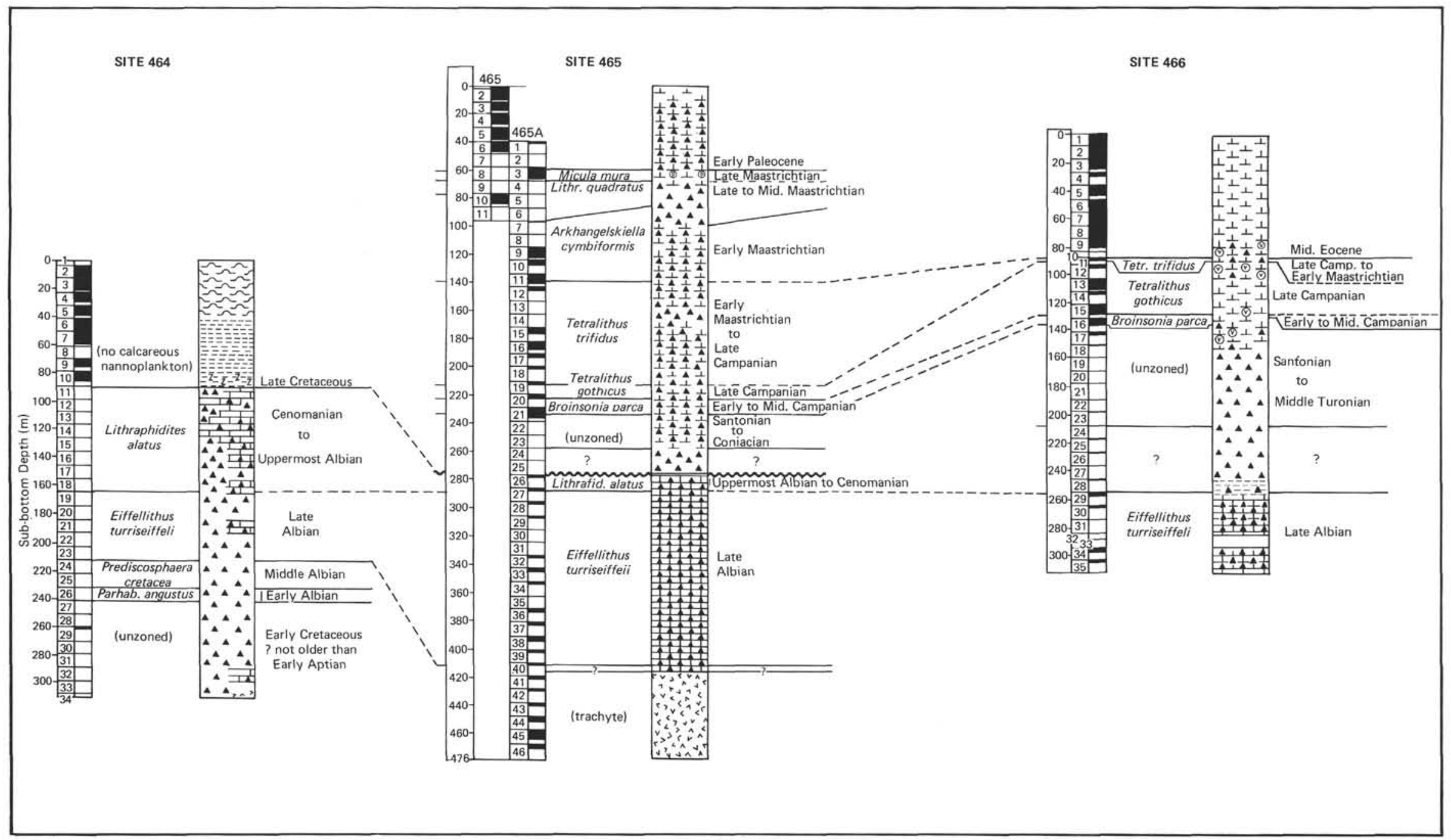

Figure 3. Comparison of Cretaceous calcareous-nannofossil zones at Sites 464 to 466 on Hess Rise. 
Table 1. Nannofossil species considered in this report, listed alphabetically according to species name.

\begin{tabular}{|c|c|c|c|c|c|}
\hline \multirow[b]{2}{*}{ Species } & \multicolumn{5}{|c|}{ Hole } \\
\hline & 463 & 464 & 465 & $465 \mathrm{~A}$ & 466 \\
\hline Corollithion achylosum (Stover) & + & & & + & + \\
\hline Tetralithus aculeus (Stradner) & + & & + & + & + \\
\hline Lithraphidites acutum Verbeek and Manivit & + & + & & + & + \\
\hline Lithraphidites alatus Thierstein & + & & & + & \\
\hline Podorhabdus albianus Black & + & + & & + & + \\
\hline Cretarhabdus angustiforatus (Black) & + & + & + & + & + \\
\hline Parhabdolithus angustus (Stradner) & + & + & + & + & + \\
\hline Parhabdolithus asper (Stradner) & + & + & & + & + \\
\hline Watznaueria barnesae (Black) & + & + & + & + & + \\
\hline Flabellites biforaminis Thierstein & + & + & & + & \\
\hline Braarudosphaera bigelowi (Gran and Braarud) & + & & & + & \\
\hline Watznaueria biporta Bukry & + & + & + & + & + \\
\hline $\begin{array}{l}\text { Watznaueria britannica (Stradner) } \\
\text { Nannoconus bucheri Bronnimann }\end{array}$ & $\begin{array}{l}+ \\
+\end{array}$ & & & & \\
\hline $\begin{array}{l}\text { Nannoconus bucheri Brönnimann } \\
\text { Lithraphidites carniolensis Deflandre }\end{array}$ & + & + & + & + & + \\
\hline Cruciellipsis chiasta (Worsley) & + & + & & + & + \\
\hline Nannoconus colomi (de Lapparent) & + & & & & \\
\hline Watznaueria communis Reinhardt & + & & & & \\
\hline Cretarhabdus conicus Bramlette and Martini & & + & + & + & + \\
\hline Biscutum constans (Gorka) & + & + & + & + & + \\
\hline Cretarhabdus coronadventis Reinhardt & + & + & & & + \\
\hline Cretarhabdus crenulatus Bramlette and Martini & & + & + & + & + \\
\hline Prediscosphaera cretacea (Arkhangelsky) & + & + & + & + & + \\
\hline Arkhangelskiella cymbiformis Vekshina & + & & + & + & + \\
\hline Microrhabdulus decoratus Deflandre & + & & + & + & + \\
\hline Zygodiscus diplogrammus (Deflandre and Fert) & + & + & + & + & + \\
\hline Cribrosphaerella ehrenbergi (Arkhangelsky) & + & + & + & + & + \\
\hline $\begin{array}{l}\text { Parhabdolithus embergeri (Noel) } \\
\text { Zygodiscus erectus (Deflandre) }\end{array}$ & + & + & + & + & + \\
\hline $\begin{array}{l}\text { Eiffellithus eximius (Stover) } \\
\text { (St) }\end{array}$ & + & & & + & + \\
\hline Lithastrinus floralis Stradner & + & + & & + & + \\
\hline Scapholithus fossilis Deflandre & & + & & & + \\
\hline Marthasterites furcatus (Deflandre) & + & & & & \\
\hline Quadrum gartneri Prins and Perch-Nielsen & + & & + & + & + \\
\hline Nannoconus globulus Bronnimann & & & & + & t \\
\hline $\begin{array}{l}\text { Tetralithus gothicus Deflandre } \\
\text { Lithastrinus grilli Stradner }\end{array}$ & + & & & + & $\begin{array}{l}+ \\
+\end{array}$ \\
\hline $\begin{array}{l}\text { Lithastrinus grilli Stradner } \\
\text { Microrhabdulus helicoideus Deflandre }\end{array}$ & + & & & & \\
\hline Micula infracretacea Thierstein & + & & & & \\
\hline Markalius inversus (Deflandre) & & & & + & \\
\hline Rucinolithus irregularis Thierstein & + & + & & & \\
\hline Stephanolithion laffittei Noel & + & & & + & + \\
\hline Chiastozygus litterarius (Gorka) & + & + & + & + & + \\
\hline Discoaster lodoensis Bramlette and Riedel & & & & $(+)$ & \\
\hline Cretarhabdus loriei Gartner & + & + & + & + & + \\
\hline $\begin{array}{l}\text { Kamptnerius magnificus Deflandre } \\
\text { Biscutum magnum Wind and Wise }\end{array}$ & + & + & + & + & + \\
\hline $\begin{array}{l}\text { Biscutum magnum Wind and Wise } \\
\text { Cyclagelosphaera margereli Noel }\end{array}$ & + & + & & + & + \\
\hline Vagalapilla matalosa (Stover) & + & & & + & + \\
\hline Conusphaera mexicana Trejo & + & & & & \\
\hline Discoaster multiradiatus Bramlette and Riedel & & & & $(+)$ & \\
\hline Micula mura (Martini) & & & $x$ & + & \\
\hline Gartmerago obliquum (Stradner) & + & & & + & + \\
\hline Tetralithus obscurus Deflandre & + & & & & \\
\hline Micrantholithus obtusus Stradner & + & & & & \\
\hline Broinsonia parca (Stradner) & & & & + & + \\
\hline Coccolithus pelagicus (Wallich) & & & & $(+)$ & $(+)$ \\
\hline Manivitella pemmatoidea (Deflandre and Manivit) & + & + & + & + & + \\
\hline Tranolithus phacelosus Stover & + & + & & + & + \\
\hline Lithraphidites quadratus Bramlette and Martini & & & + & + & \\
\hline Parhabdolithus regularis (Gorka) & + & & + & + & + \\
\hline Cretarhabdus schizobrachiatus (Gartner) & & & + & + & + \\
\hline Lithastrinus septentrionalis Stradner & + & & & & \\
\hline Corollithion signum Stradner & + & + & & + & + \\
\hline Prediscosphaera spinosa (Bramlette and Martini) & & & + & + & + \\
\hline Parhabdolithus splendens Deflandre & & & + & + & + \\
\hline Micula staurophora (Gardet) & + & & + & + & + \\
\hline Micula staurophora concava (Stradner) & & & & + & \\
\hline Microrhabdulus stradneri Bramlette and Martini & + & & + & + & \\
\hline Vagalapilla stradneri (Rood, Hay, and Barnard) & + & & + & + & + \\
\hline Discoaster surculus Martini and Bramlette & & & & & $(+)$ \\
\hline Cruciplacolithus tenuis (Stradner) & & & & $(+)$ & \\
\hline Eiffellithus trabeculatus (Gorka) & & & & + & + \\
\hline Tetralithus trifidus (Stradner) & + & & & + & + \\
\hline Nannoconus truitti Brönnimann & + & & & & \\
\hline Eiffellithus turriseiffeli (Deflandre and Fert) & + & + & + & + & + \\
\hline Biscutum sp. & & + & & + & + \\
\hline $\begin{array}{l}\text { Braarudosphaera sp. } \\
\text { Broinsonia } \mathrm{sp} \text {. }\end{array}$ & + & & & + & + \\
\hline Cretarhabdus sp. & + & + & + & + & \\
\hline Cribrosphaerella sp. & & & & + & \\
\hline Discoaster sp. & & $(+)$ & & $(+)$ & \\
\hline Lithraphidites sp. & & + & & & \\
\hline $\begin{array}{l}\text { Microrhabdulus sp. } \\
\text { Nannoconus sp. }\end{array}$ & + & + & & + & + \\
\hline $\begin{array}{l}\text { Nannoconus sp. } \\
\text { Scapholithus sp. }\end{array}$ & & & & + & \\
\hline Tranolithus sp. & & & & & + \\
\hline Vagalapilla sp. & + & & & & \\
\hline Watznaueria sp. & + & 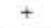 & & & \\
\hline
\end{tabular}

Note: $+=$ present; $x=$ cf.; $(+)=$ down-hole contamination.

\section{Parhabdolithus angustus Zone}

Author: Manivit (1971), modified by Thierstein (1973).

Definition: Interval from the first occurrence of Parhabdolithus angustus (Stradner) and (or) Lithastrinus floralis Stradner to the first occurrence of Prediscosphaera cretacea (Arkhangelsky).

Age: Late Aptian to early Albian

Important common species: Parhabdolithus angustus (Stradner), Lithastrinus floralis Stradner, Nannoconus bucheri Brönnimann, Micrantholithus obtusus Stradner, Chiastozygus litterarius (Gorka), and Rucinolithus irregularis Thierstein.

Remarks: This zone was identified in Holes 463 and 464, but in Hole 464 the Parhabdolithus angustus Zone is limited to Core 27. In Hole 463 , it is possible to subdivide this zonal interval into two parts. The upper part is determined at the top by the first occurrence of Prediscosphaera cretacea (Arkhangelsky), and at the base by the last occurrence of Nannoconus bucheri Brönnimann. The lower part of this zonal interval is characterized by the occurrence together of Lithastrinus floralis Stradner and Nannoconus bucheri Brönnimann.

\section{Chiastozygus litterarius Zone}

Author: Thierstein (1971).

Definition: Interval from the last occurrence of Nannoconus colomi (de Lapparent) and (or) the first occurrence of Chiastozygus litterarius (Gorka) to the first occurrence of Parhabdolithus angustus (Stradner) and (or) Lithastrinus floralis Stradner.

Age: Early Aptian.

Important common species: Chiastozygus litterarius (Gorka), Micrantholithus obtusus Stradner, and Rucinolithus irregularis Thierstein.

Remarks: This zone was cored only in Hole 463. In this paper the first occurrence of Chiastozygus litterarius (Gorka) and the last occurrence of Nannoconus colomi (de Lapparent) are used to define the lower boundary of this zone. But it is difficult to recognize the species Chiastozygus litterarius (Gorka). In this zonal interval of Hole 463 , this species is rare and not well preserved.

\section{Micrantholithus obtusus Zone}

Author: Thierstein (1971).

Definition: Interval from the last occurrence of Calcicalathina oblongata (Worsley) to the last occurrence of Nannoconus colomi (de Lapparent) and (or) the first occurrence of Chiastozygus litterarius (Gorka).

Age: Barremian.

Important common species: Nannoconus colomi (de Lapparent) and Micrantholithus obtusus Stradner.

Remarks: The Micrantholithus obtusus Zone is the oldest Cretaceous zone recorded during Leg 62 . This zone is only recognized in Hole 463 , but it is impossible to define the lower boundary of this zonal interval, because Calcicalathina oblongata (Worsley) is missing. The absence of this resistant species strongly suggests that drilling at Site 463 terminated in sediments of Barremian age.

\section{DISTRIBUTION OF CALCAREOUS NANNOFOSSILS}

\section{Site 463 \\ $\left(21^{\circ} 21.01^{\prime} \mathrm{N}, 174^{\circ} 40.07^{\prime} \mathrm{E}\right.$, depth $\left.2525 \mathrm{~m}\right)$}

Coring at Site 463 , on the western Mid-Pacific Mountains was continuous to a sub-bottom depth of 822.5 meters (Fig. 1). The recovered sediments are divided into four lithologic units. The youngest lithologic unit consists of a 405-meter sequence of nannoplankton and foraminifer chalk, ooze, and limestone which includes a Cretaceous section of late Albian to early Maastrichtian age.

The second lithologic unit is 136 meters thick and consists of cyclic alternations of green and gray and green and pink pelagic limestones with abundant chert. These limestones range in age from late Aptian through middle Aptian. 
The underlying unit, 45 meters thick, consists of carbonaceous limestone, black claystone and marlstone, and volcanic ash, of early Aptian age.

The oldest unit consists of interbedded pelagic and clastic limestones; it is more than 190 meters thick and belongs to the Barremian to early Aptian.

Abundant to rare but poorly preserved nannofossil assemblages were found throughout the Cretaceous section (Table 2, back pocket; Fig. 4). A sequence of 11 nannoplankton zones or zonal intervals has been recognized in what appears to be continuous deposition from Barremian to early Maastrichtian. Certain intervals are less distinct, because of the lack of index species important for zonation, particularly in sediments of Turonian to Campanian age.

Samples from the interval from Core 7, Section 3, 30 $\mathrm{cm}$ to $16, \mathrm{CC}$ contain abundant coccoliths, including Arkhangelskiella cymbiformis Vekshina. The assemblages lack Lithraphidites quadratus Bramlette and Martini, Micula mura (Martini), and Nephrolithus frequens Gorka. These are all index species of late Maastrichtian age. Tetralithus trifidus (Stradner), an index species of late Campanian to early Maastrichtian age, is also missing. Thus, this interval is assigned to the Arkhangelskiella cymbiformis Zone of early Maastrichtian age.

The assemblages from Cores 17 to 22 have abundant nannofossils, with Tetralithus trifidus (Stradner) and Tetralithus gothicus Deflandre. The total range of the index species defines the Tetralithus trifidus Zone of late Campanian to early Maastrichtian age.

Nannofossils are common to abundant in this stratigraphic interval (Samples 23-1, 51-52 cm to 25-2, 62-63 $\mathrm{cm}$ ), but their preservation is poor. The first occurrence of Tetralithus gothicus Deflandre to the first occurrence of Tetralithus trifidus (Stradner) delimits the Tetralithus gothicus Zone, of late Campanian age.

Common to abundant assemblages of Coniacian to late Campanian age $(25, \mathrm{CC}$ to $30, \mathrm{CC})$ are characterized by the lack of index species. The upper limit of this zonal interval is determined by the first occurrence of Tetralithus gothicus Deflandre, and the lower limit by the last occurrence of Corollithion achylosum (Stover), the range of which according to Thierstein (1976) should extend to the top of the Turonian.

In the next zonal interval $(31, \mathrm{CC}$ to $37, \mathrm{CC})$ marker species are also missing. The top of this zonal interval (Turonian) is determined by the last occurrence of Corollithion achylosum (Stover), and the lower limit has been assessed by the last occurrence of Lithraphidites acutum. Verbeek and Manivit.

Common to abundant assemblages of coccoliths were recovered in Samples 38-1, 29-30 cm to 50-1, 122-124 $\mathrm{cm}$. The first occurrence of Lithraphidites alatus Thierstein and the last occurrence of Lithraphidites acutum Verbeek and Manivit are used to define the Lithraphidites alatus Zone of uppermost Albian to Cenomanian age.

The common nannoflora assemblages from the interval 50,CC to 52,CC with Eiffellithus turriseiffeli (Deflandre and Fert) but without Lithraphidites alatus
Thierstein and Lithraphidites acutum Verbeek and Manivit, indicate the Eiffellithus turriseiffeli Zone of late Albian age.

Samples from the interval $53-1,122-124 \mathrm{~cm}$ to $55-1$, $22-23 \mathrm{~cm}$ contain common to abundant, but poorly preserved calcareous nannofossils. This short interval is limited at the top by the first occurrence of Eiffellithus turriseiffeli (Deflandre and Fert) and at the base by the first occurrence of Prediscosphaera cretacea (Arkhangelsky). It belongs to the Prediscosphaera cretacea Zone, of middle Albian age.

The Parhabdolithus angustus Zone of late Aptian to early Albian age was identified in Samples 58,CC to $65-2,7-8 \mathrm{~cm}$. It is possible to divide this zonal interval into two stratigraphic units: an upper one (early Albian) and a lower one (late Aptian). The top of the upper one is determined by the first occurrence of Prediscosphaera cretacea (Arkhangelsky), and the base by the last occurrence of Nannoconus bucheri Bronnimann. The lower unit of the Parhabdolithus angustus Zone can be characterized by the occurrence together of Lithastrinus floralis Stradner and Nannoconus bucheri Brönnimann.

Generally rare and poorly preserved nannofossils occur in the interval $65, \mathrm{CC}$ to $78-1,87-88 \mathrm{~cm}$, with Chiastozygus litterarius (Gorka) and Rucinolithus irregularis Thierstein. Since Lithastrinus floralis Stradner is missing, this interval represents the Chiastozygus litterarius Zone, of Early Aptian age.

Nannofossils in Samples $78-1,87-88 \mathrm{~cm}$ to $92, \mathrm{CC}$ (last core) are generally very rare. Calcicalathina oblongata (Worsley) and Cruciellipsis cuvillieri (Manivit), whose last occurrences are listed as early Barremian and late Hauterivian, respectively (Thierstein, 1976), have not been found. Thus, this interval with Micrantholithus obtusus Stradner is assigned to the Micrantholithus obtusus Zone, of Barremian age. The absence of resistant Calcicalathina oblongata (Worsley) strongly suggests that Site 463 terminated in sediments of Barremian age.

\section{Site 464 \\ $\left(39^{\circ} 51.64^{\prime} \mathrm{N}, 173^{\circ} 53.33^{\prime} \mathrm{E}\right.$, depth $\left.4637 \mathrm{~m}\right)$}

Site 464 is on northern Hess Rise (Fig. 1). The total depth drilled was 308.5 meters. Abundant to rare (predominantly abundant), but poorly preserved calcareous nannoplankton assemblages were found throughout the Lower Cretaceous and lowermost Upper Cretaceous. A sequence of five nannoplankton zones or zonal intervals has been recognized within the lithologic unit of redbrown chert, chalk, and marlstone (Table 3; Fig. 5). This lithologic unit overlies basalt.

At the top of the Cretaceous sediments (Sample 11-1, $68-69 \mathrm{~cm}$ to $18, \mathrm{CC}, 17-18 \mathrm{~cm}$ ), rich assemblages of poorly to moderately preserved nannofossils occur with the index species Lithraphidites acutum Verbeek and Manivit. These assemblages suggest for this part of the profile a latest Albian to Cenomanian age (Lithraphidites alatus Zone).

In Samples $18, \mathrm{CC}$ to $24-1,15-16 \mathrm{~cm}$, abundant but poorly preserved late Albian coccolith assemblages occur, including the zone marker Eiffellithus turriseiffeli 


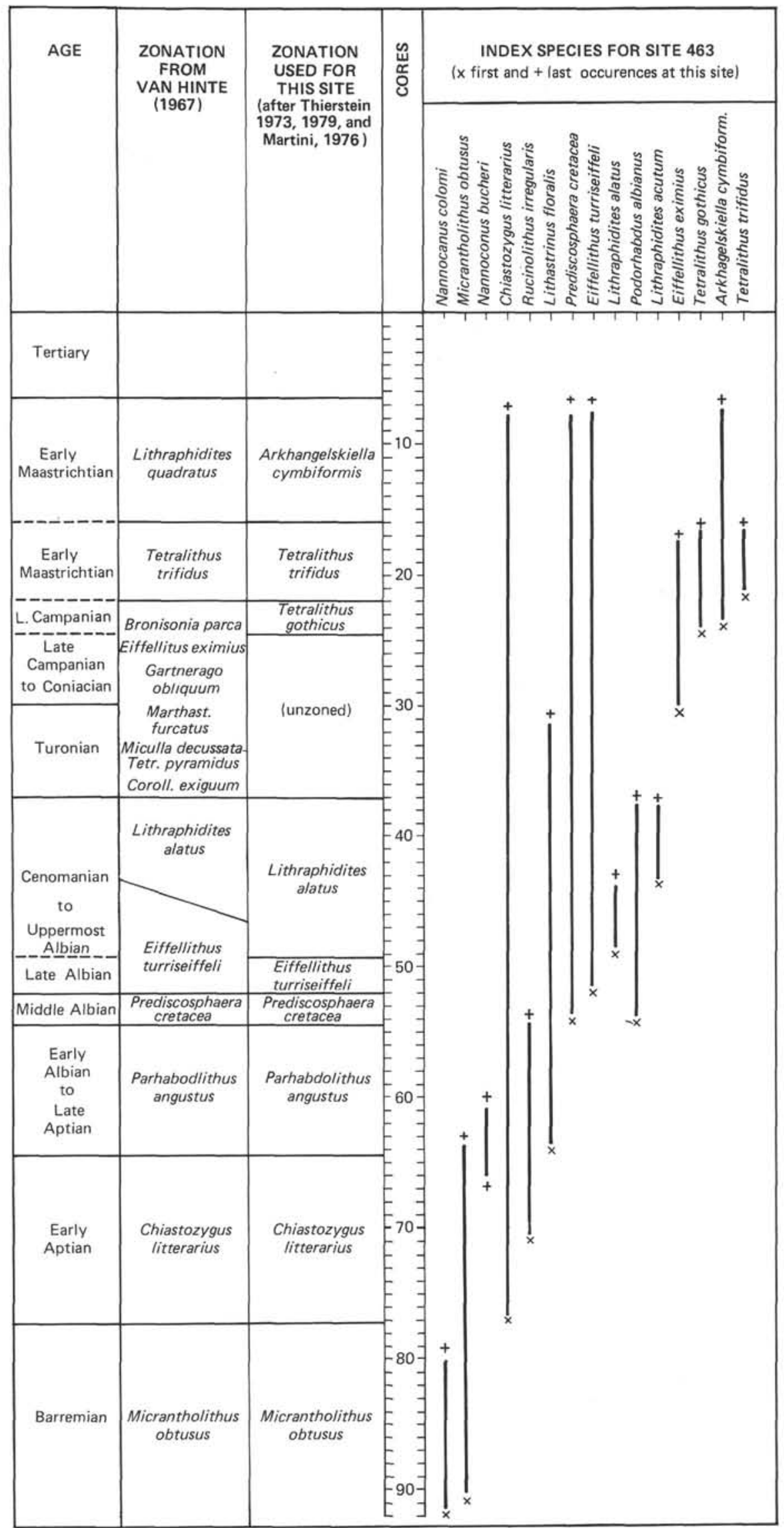

Figure 4. Ranges of index species at Site 463 and correlation of the zonation used with that according to van Hinte (1976). 
Table 3. Distribution and abundance of calcareous-nannoplankton species in the Cretaceous sediments of Site 464.

\begin{tabular}{|c|c|c|c|c|c|c|c|c|c|c|c|c|c|c|c|c|c|c|}
\hline $\begin{array}{c}\text { Sample } \\
\text { (interval } \\
\text { in } \mathrm{cm} \text { ) }\end{array}$ & $\begin{array}{c}\text { Sub- } \\
\text { bottom } \\
\text { Depth } \\
\text { (m) }\end{array}$ & 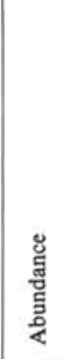 & 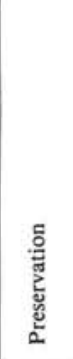 & \& & 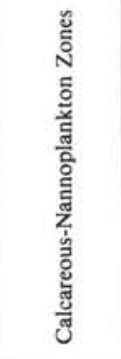 & 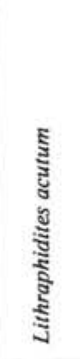 & 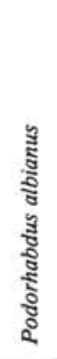 & 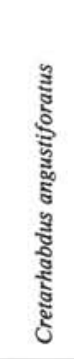 & 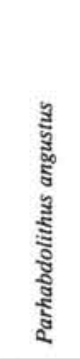 & 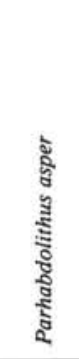 & 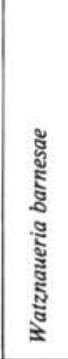 & 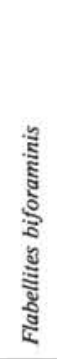 & 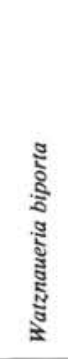 & 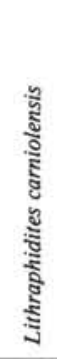 & 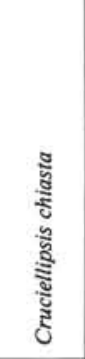 & 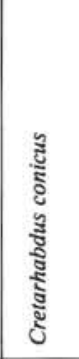 & 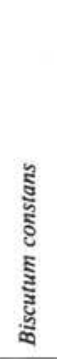 & 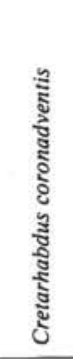 \\
\hline $\begin{array}{c}\text { 464-11-1, } 68-69 \\
11-1,140 \\
12-1,21-22 \\
12-1,49-50 \\
12, \mathrm{CC} \\
13, \mathrm{CC}, 13-14 \\
13, \mathrm{CC}, \text { base } \\
\text { 14,CC, } 41-42 \\
\text { (bottom) } \\
\text { 15,CC, } 27-28 \\
\text { 15,CC, base } \\
16, \mathrm{CC}, 8-9 \\
16, \mathrm{CC}, \text { base } \\
17, \mathrm{CC}, 17-18 \\
\text { 17,CC, base } \\
\text { 18,CC, } 22-23 \\
\end{array}$ & $\begin{array}{r}89.68 \\
90.40 \\
98.71 \\
98.99 \\
108.00 \\
117.49 \\
117.50 \\
127.00 \\
136.45 \\
136.50 \\
145.95 \\
146.00 \\
155.35 \\
155.50 \\
164.90 \\
\end{array}$ & $\begin{array}{l}\text { F } \\
\text { A } \\
\text { F } \\
\text { A } \\
\text { A } \\
\text { A } \\
\text { A } \\
\text { F } \\
\text { A } \\
\text { A } \\
\text { C } \\
\text { F } \\
\text { A } \\
\text { A } \\
\text { C }\end{array}$ & $\begin{array}{c}\mathrm{P} \\
\mathrm{P} \\
\mathrm{P} \\
\mathrm{M} \\
\mathrm{P} \\
\mathrm{M}-\mathrm{G} \\
\mathrm{P}-\mathrm{M} \\
\mathrm{P} \\
\mathrm{M} \\
\mathrm{M} \\
\mathrm{P}-\mathrm{M} \\
\mathrm{P} \\
\mathrm{P} \\
\mathrm{M} \\
\mathrm{M} \\
\mathrm{P}\end{array}$ & $\begin{array}{l}\text { Ceno- } \\
\text { manian } \\
\text { to Upper- } \\
\text { most } \\
\text { Albian }\end{array}$ & $\begin{array}{c}\text { Lithra- } \\
\text { phidites } \\
\text { alatus }\end{array}$ & $\begin{array}{c}\mathrm{C} \\
\mathrm{VR} / ? \\
\mathrm{~F} \\
\mathrm{VR}\end{array}$ & $\begin{array}{l}\text { C } \\
\text { VR } \\
\text { VR } \\
\text { C } \\
\text { C } \\
\text { R }\end{array}$ & & VR & $\begin{array}{l}\mathrm{R} / \mathrm{X} \\
\mathrm{VR} / \mathrm{X}\end{array}$ & $\begin{array}{l}\text { F } \\
\text { C } \\
\text { C } \\
\text { A } \\
\text { A } \\
\text { A } \\
\text { A } \\
\text { A } \\
\\
\text { A } \\
\mathrm{A} \\
\mathrm{A} \\
\mathrm{C} \\
\mathrm{A} \\
\mathrm{A} \\
\mathrm{A}\end{array}$ & $\begin{array}{c}C \\
\text { VR } \\
C\end{array}$ & $\begin{array}{c}\mathrm{R} \\
\mathrm{F} \\
\mathrm{VR} \\
\mathrm{F}\end{array}$ & $\begin{array}{l}\mathrm{F} \\
\mathrm{C} \\
\mathrm{F} \\
\mathrm{C} \\
\mathrm{F} \\
\\
\mathrm{F} \\
\mathrm{F} \\
\mathrm{F} \\
\mathrm{C} \\
\mathrm{C} \\
\mathrm{F}\end{array}$ & & & $\begin{array}{l}\text { C } \\
\text { VR } \\
\text { C } \\
\text { A } \\
\text { C }\end{array}$ & $\mathrm{VR} / \mathrm{X}$ \\
\hline $\begin{array}{l}18, \mathrm{CC}, \text { base } \\
19, \mathrm{CC} \\
20-1 \\
21-1,3-4 \\
21-1,12-13 \\
22, \mathrm{CC} \\
23-1,5 \\
23-1,40 \\
23-1,60 \\
24-1,15-16 \\
\end{array}$ & $\begin{array}{r}165.00 \\
174.50 \\
? 184.00 \\
184.03 \\
184.12 \\
203.00 \\
203.05 \\
203: 40 \\
203.60 \\
212.65 \\
\end{array}$ & $\begin{array}{c}\text { C-A } \\
\text { A } \\
\text { A } \\
\text { R } \\
\text { F-C } \\
\text { C } \\
\text { A } \\
\text { A } \\
\text { A } \\
\text { A }\end{array}$ & \begin{tabular}{|c|}
$\mathrm{P}$ \\
$\mathrm{P}$ \\
$\mathrm{P}$ \\
$\mathrm{P}$ \\
$\mathrm{P}$ \\
$\mathrm{P}$ \\
$\mathrm{P}$ \\
$\mathrm{P}$ \\
$\mathrm{P}$ \\
$\mathrm{P}-\mathrm{M}$
\end{tabular} & $\begin{array}{c}\text { Late } \\
\text { Albian }\end{array}$ & $\begin{array}{l}\text { Eiffel- } \\
\text { lithus } \\
\text { turris- } \\
\text { eiffeli }\end{array}$ & & $\begin{array}{c}\mathrm{R} \\
\mathrm{VR}\end{array}$ & & $\begin{array}{c}\mathrm{R} \\
\mathrm{VR} / \mathrm{X}\end{array}$ & $\begin{array}{c}\mathrm{VR} / \mathrm{X} \\
\mathrm{C} \\
\end{array}$ & $\begin{array}{l}\mathrm{A} \\
\mathrm{C} \\
\mathrm{A} \\
\\
\mathrm{A} \\
\mathrm{A} \\
\mathrm{A} \\
\mathrm{A} \\
\mathrm{A} \\
\mathrm{A}\end{array}$ & $\mathrm{C}$ & $\begin{array}{l}\text { VR } \\
\text { VR } \\
\text { VR } \\
\text { VR } \\
\text { R } \\
\text { C } \\
\text { C } \\
\text { R } \\
\text { C } \\
\end{array}$ & $\begin{array}{l}\text { C } \\
\text { F } \\
\text { C } \\
\text { VR } \\
\text { C } \\
\text { C } \\
\text { C } \\
\text { C } \\
\text { A } \\
\end{array}$ & $\begin{array}{c}\mathrm{VR} / \mathrm{X} \\
\mathrm{VR} / \mathrm{X} \\
\mathrm{C} \\
\end{array}$ & VR/? & $\begin{array}{l}\text { C } \\
\text { C } \\
\text { A } \\
\\
\text { F } \\
\text { A } \\
\text { C } \\
\text { A } \\
\text { A } \\
\text { A }\end{array}$ & VR \\
\hline $\begin{array}{l}25, C C \\
26-1,75-76\end{array}$ & $\begin{array}{l}231.50 \\
232.25 \\
\end{array}$ & $\begin{array}{c}\mathrm{F}-\mathrm{C} \\
\mathrm{C}\end{array}$ & $\begin{array}{l}\mathrm{P} \\
\mathrm{P} \\
\end{array}$ & $\begin{array}{c}\text { Mid. } \\
\text { Albian }\end{array}$ & $\begin{array}{l}\text { Predisc. } \\
\text { cretacea }\end{array}$ & & $R$ & $\mathrm{VR} / \mathrm{X}$ & $\mathrm{F}$ & & $\begin{array}{l}\text { A } \\
\text { A }\end{array}$ & & $\begin{array}{l}\mathrm{C} \\
\mathrm{F}\end{array}$ & $\begin{array}{l}\mathrm{F} \\
\mathrm{C}\end{array}$ & $F / X$ & VR & $\begin{array}{l}\mathrm{F} \\
\mathrm{F}\end{array}$ & $\mathrm{R}$ \\
\hline $27-1,25-26$ & 241.25 & A & $\mathrm{M}$ & E. Alb. & P. angus & & & & $\mathrm{F}$ & $\mathrm{F}$ & $\mathrm{A}$ & & $\mathrm{C}$ & $\mathrm{C}$ & & & $\mathrm{C}$ & $\mathrm{F}$ \\
\hline $\begin{array}{l}28-1,25-26 \\
29-1,65-66 \\
30-1, \text { top } \\
31-1,3-4 \\
31-1,27-28 \\
32-1,18-19\end{array}$ & $\begin{array}{l}250.75 \\
260.65 \\
269.50 \\
279.03 \\
279.27 \\
288.68\end{array}$ & \begin{tabular}{|c} 
(ba \\
$\mathrm{C}-\mathrm{A}$ \\
$\mathrm{R}$ \\
$\mathrm{R}$ \\
$\mathrm{A}$ \\
$\mathrm{C}-\mathrm{A}$
\end{tabular} & $\begin{array}{r}\text { ren) } \\
P \\
P \\
P \\
P \\
P\end{array}$ & $\begin{array}{c}\text { Early } \\
\text { Cretaceous } \\
\text { ?not } \\
\text { older } \\
\text { than E. } \\
\text { Aptian }\end{array}$ & (unzoned) & & & & & & $\begin{array}{c}\text { F/X } \\
\text { C } \\
\text { VR } \\
\text { A } \\
\text { A }\end{array}$ & & $\begin{array}{c}\mathrm{C} \\
\mathrm{R} / \mathrm{X} \\
\mathrm{R} / \mathrm{X} \\
\mathrm{R} \\
\mathrm{C}\end{array}$ & $\begin{array}{c}\text { VR } \\
\text { C }\end{array}$ & & & & \\
\hline
\end{tabular}

Note: Symbols as in Table 2.

(Deflandre and Fert), but without Lithraphidites acutum Verbeek and Manivit. This assemblage indicates the Eiffellithus turriseiffeli Zone.

Poorly preserved and predominantly common assemblages of calcareous nannoplankton in Samples 25,CC to 26-1, 75-76 $\mathrm{cm}$, with Prediscosphaera cretacea (Arkhangelsky), were assigned to the middle Albian (Prediscosphaera cretacea Zone), as they are lacking Eiffellithus turriseiffeli (Deflandre and Fert).

Prediscosphaera cretacea (Arkhangelsky) is missing in Sample 27-1, 25-26 cm, and the presence of the zone markers Parhabdolithus angustus (Stradner) and Lithastrinus floralis Stradner assign this sample to the early Albian (Parhabdolithus angustus Zone).

Core 28 is barren of calcareous nannofossils. Cores 29 to 32 generally contain abundant nannofossils which are very poorly preserved. As a result of strong dissolution, all index species are missing, and only solutionresistant species such as Watznaueria barnesae (Black), Manivitella pemmatoidea (Deflandre and Manivit), Parhabdolithus embergeri (Noel), and Lithraphidites carniolensis Deflandre are present. The absence of index species makes it impossible to determine an exact stratigraphic interval within the Lower Cretaceous. Probably this oldest stratigraphic interval of the Cretaceous at Site 464 is not older than Aptian, as Nannoconus colomi (de Lapparent), a very solution-resistant species which has its last occurrence at the top of the Barremian, is not present.

\section{Site 465 \\ $\left(33^{\circ} 49.23^{\prime} \mathrm{N}, 1^{\circ} 8^{\circ} 55.14^{\prime} \mathrm{E}\right.$, depth $\left.2161 \mathrm{~m}\right)$}

Two holes were drilled at Site 465 , on southern Hess Rise (Fig. 1). The total depth drilled in Hole 465 was 96 meters, and in Hole 465A 411.7 meters.

Predominantly abundant and moderately well-preserved nannofossils were found throughout the Upper Cretaceous section. Poorly preserved calcareous nannofossils were commonly recovered from the Lower Cretaceous. A sequence of 9 nannoplankton zones or zonal intervals has been recognized (Tables 4 and 5; Fig. 6). The Cretaceous sediments are found in both sedimentary lithologic units of this site. The youngest sedimentary unit (late Turonian to Maastrichtian) in- 
Table 3. (Continued).

\begin{tabular}{|c|c|c|c|c|c|c|c|c|c|c|c|c|c|c|c|c|c|c|c|c|c|}
\hline 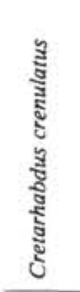 & 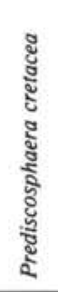 & 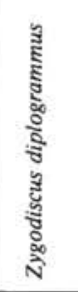 & 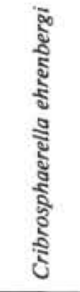 & 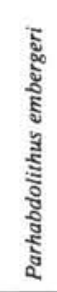 & 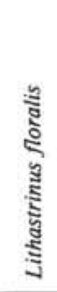 & 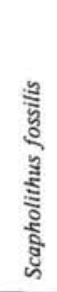 & 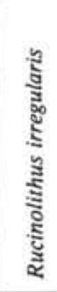 & 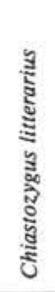 & 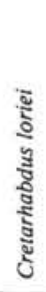 & 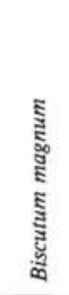 & 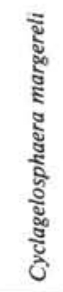 & 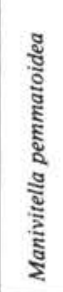 & 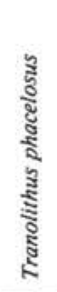 & 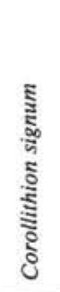 & 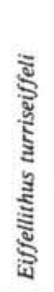 & 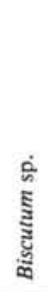 & 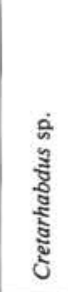 & 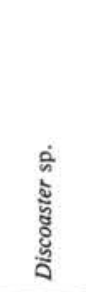 & 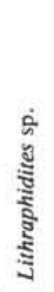 & 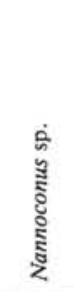 & 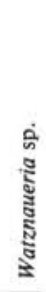 \\
\hline $\begin{array}{c}\text { VR } \\
\text { R } \\
\text { VR }\end{array}$ & $\begin{array}{l}\mathrm{F} \\
\mathrm{C} \\
\mathrm{F} \\
\mathrm{A} \\
\mathrm{A} \\
\mathrm{A} \\
\mathrm{C} \\
\mathrm{F}\end{array}$ & $\begin{array}{c}\mathrm{R} / \mathrm{X} \\
\mathrm{C} \\
\mathrm{C} \\
\mathrm{C} \\
\mathrm{F} \\
\mathrm{F}\end{array}$ & & $\begin{array}{l}\mathrm{F} \\
\mathrm{F} \\
\mathrm{F} \\
\mathrm{R} \\
\mathrm{R}\end{array}$ & $\begin{array}{l}\mathrm{R} \\
\mathrm{C} \\
\mathrm{F} \\
\mathrm{C} \\
\mathrm{C} \\
\mathrm{A} \\
\mathrm{C}\end{array}$ & & & $\begin{array}{c}\text { VR } \\
\text { F } \\
\text { VR }\end{array}$ & & & & $\begin{array}{l}\text { F } \\
\text { C } \\
\text { C } \\
\text { C } \\
\text { C } \\
\text { A } \\
\text { A } \\
\text { C }\end{array}$ & VR & . & $\begin{array}{l}\mathrm{R} \\
\mathrm{F} \\
\mathrm{F} \\
\mathrm{R} \\
\mathrm{C} \\
\mathrm{C}\end{array}$ & & $\begin{array}{l}\mathrm{F} \\
\mathrm{R} \\
\mathrm{C} \\
\mathrm{R} \\
\mathrm{A} \\
\mathrm{C} \\
\mathrm{F}\end{array}$ & & & & $\begin{array}{l}\mathrm{F} \\
\mathrm{C} \\
\mathrm{C} \\
\mathrm{A} \\
\mathrm{C} \\
\mathrm{A} \\
\mathrm{R} \\
\mathrm{A}\end{array}$ \\
\hline $\begin{array}{c}\mathrm{C} \\
\mathrm{C} \\
\mathrm{C} \\
\mathrm{VR} \\
\mathrm{C} \\
\mathrm{F} \\
\mathrm{F} \\
\end{array}$ & $\begin{array}{l}\text { VR } \\
\text { VR } \\
\text { C } \\
\text { F } \\
\text { F }\end{array}$ & $\begin{array}{l}\mathrm{C} \\
\mathrm{C} \\
\mathrm{C}\end{array}$ & & $\begin{array}{l}\mathrm{C} \\
\mathrm{F} \\
\mathrm{C} \\
\mathrm{F} \\
\mathrm{F} \\
\mathrm{F}\end{array}$ & $\begin{array}{c}\text { F } \\
C \\
\text { R } \\
\text { VR } \\
\text { F }\end{array}$ & $F / X$ & & $\begin{array}{c}\mathrm{C} \\
\mathrm{F} \\
\text { VR }\end{array}$ & & & & $\begin{array}{l}\text { A } \\
\text { A } \\
\text { C } \\
\text { C } \\
\text { C } \\
\text { A } \\
\text { F }\end{array}$ & $\begin{array}{c}\mathrm{R} \\
\mathrm{VR} \\
\mathrm{C}\end{array}$ & VR & $\begin{array}{l}\mathrm{C} \\
\mathrm{C} \\
\mathrm{C} \\
\mathrm{C} \\
\mathrm{C} \\
\mathrm{A} \\
\mathrm{C}\end{array}$ & & $\begin{array}{c}\text { C } \\
C \\
C \\
\text { VR } \\
C \\
C \\
F \\
\end{array}$ & $\mathrm{~F} /(+)$ & & & $\begin{array}{l}\text { A } \\
\text { C } \\
\text { C } \\
\text { C } \\
\text { A } \\
\text { A } \\
\text { A }\end{array}$ \\
\hline $\begin{array}{c}\text { VR } \\
\text { R } \\
C \\
F \\
\text { F } / X \\
\end{array}$ & $\begin{array}{l}\mathrm{F} \\
\mathrm{R} \\
\mathrm{C} \\
\mathrm{C} \\
\mathrm{F}\end{array}$ & $\begin{array}{l}\mathrm{R} \\
\mathrm{F} \\
\mathrm{C} \\
\mathrm{C} \\
\mathrm{C} \\
\mathrm{C} \\
\end{array}$ & $\mathrm{VR} / \mathrm{X}$ & $\begin{array}{c}\text { VR } \\
\text { F } \\
F\end{array}$ & $\begin{array}{l}\text { VR } \\
\text { VR }\end{array}$ & & & $\begin{array}{c}\text { VR } \\
\text { F } \\
F \\
F \\
\text { C }\end{array}$ & & & & $\begin{array}{l}\text { C } \\
\text { F } \\
\text { C } \\
\text { F } \\
\text { C } \\
\text { C } \\
\text { R } \\
\text { F } \\
\text { C } \\
\text { R }\end{array}$ & $\begin{array}{l}\mathrm{F} \\
\mathrm{C}\end{array}$ & & $\begin{array}{l}\mathrm{F} \\
\mathrm{C} \\
\mathrm{C} \\
\mathrm{A} \\
\mathrm{A} \\
\mathrm{A}\end{array}$ & C & $\begin{array}{l}\mathrm{C} \\
\mathrm{F} \\
\mathrm{F} \\
\mathrm{F} \\
\mathrm{C} \\
\mathrm{C}\end{array}$ & & & & $\begin{array}{l}\text { A } \\
\text { F } \\
\text { C } \\
\text { C } \\
\text { A } \\
\text { C } \\
\text { C } \\
\text { F } \\
\text { C } \\
\text { C }\end{array}$ \\
\hline $\begin{array}{l}\mathrm{F} \\
\mathrm{C}\end{array}$ & $R$ & & & F & & & F & C & & & & $\begin{array}{l}\mathrm{F} \\
\mathrm{C}\end{array}$ & & & & & VR & & & & $\begin{array}{l}\text { A } \\
\text { C }\end{array}$ \\
\hline $\mathrm{F}$ & & $\mathrm{F}$ & & $\mathrm{F}$ & VR & & $\mathrm{F}$ & C & $\mathrm{F}$ & $F / X$ & $F / X$ & $\mathrm{C}$ & $\mathrm{F}$ & & & & $\mathbf{R}$ & & & & A \\
\hline $\mathrm{VR} / ?$ & & & & $\begin{array}{c}\mathrm{C} \\
\mathrm{F} \\
\mathrm{VR} \\
\mathrm{C}\end{array}$ & & & VR & & & & & $\begin{array}{c}\mathrm{C} \\
\text { VR } \\
\text { VR } \\
\text { VR }\end{array}$ & & & & & $\mathrm{VR} / ?$ & & F & VR/? & $\begin{array}{l}\mathrm{C} \\
\mathrm{C} \\
\mathrm{C}\end{array}$ \\
\hline
\end{tabular}

cludes the Micula mura Zone, Lithraphidites quadratus Zone, Arkhangelskiella cymbiformis Zone, Tetralithus trifidus Zone, Tetralithus gothicus Zone, Broinsonia parca Zone, and an unzoned interval (Coniacian to Santonian). It consists of nannofossil ooze and foraminifer ooze, with interlayered chert of Coniacian to Maastrichtian age. The oldest unit, of Albian to Cenomanian age (Lithraphidites alatus Zone and Eiffellithus turriseiffeli Zone), consists of olive-gray laminated limestone (136 $\mathrm{m})$, which overlies basalt.

The first Cretaceous sediments with abundant and moderately to well-preserved calcareous nannoplankton were encountered at the depth of 62.20 meters, in Sample $465 \mathrm{~A}-3,120 \mathrm{~cm}$, and the presence of Micula mura (Martini) indicates the late Maastrichtian. The range of this species determines the youngest zone (Micula mura Zone) of the Cretaceous. A success of Hole $465 \mathrm{~A}$ was the recovery of an apparently continuous sedimentary sequence across the Cretaceous/Tertiary boundary, with a well-preserved assemblage of the Globigerina eugubina Zone (foraminifer) in the lowermost Paleo- cene. This stratigraphic interval was not identified in Hole 465.

The recovered Cretaceous sediments of Hole 465 (in Samples $9, \mathrm{CC}$ to $11, \mathrm{CC}$ ) belong only to the Lithraphidites quadratus Zone, of middle to late Maastrichtian age. This zone is present also in Hole 465A, but only in Core 4. According to the correlation of both holes, this zone should be present also in Core $465 \mathrm{~A}-5$, and partly in Core 465A-6. However, between samples 4A,CC and $6 \mathrm{~A}, \mathrm{CC}$, no samples are available (only chert).

The abundant coccolith assemblages from the interval $465 \mathrm{~A}-6, \mathrm{CC}$ to $465 \mathrm{~A}-11-5,8-9 \mathrm{~cm}$ include the index species Arkhangelskiella cymbiformis Vekshina. The assemblages lack Lithraphidites quadratus Bramlette and Martini (index species of the late Maastrichtian) and Tetralithus trifidus (Stradner) (index species of late Campanian to earliest Maastrichtian), and are assigned to the Arkhangelskiella cymbiformis Zone, of early Maastrichtian age.

The late Campanian to early Maastrichtian (Samples $465 \mathrm{~A}-11, \mathrm{CC}$ to $465 \mathrm{~A}-19, \mathrm{CC})$ are represented by assem- 
P. ČEPEK

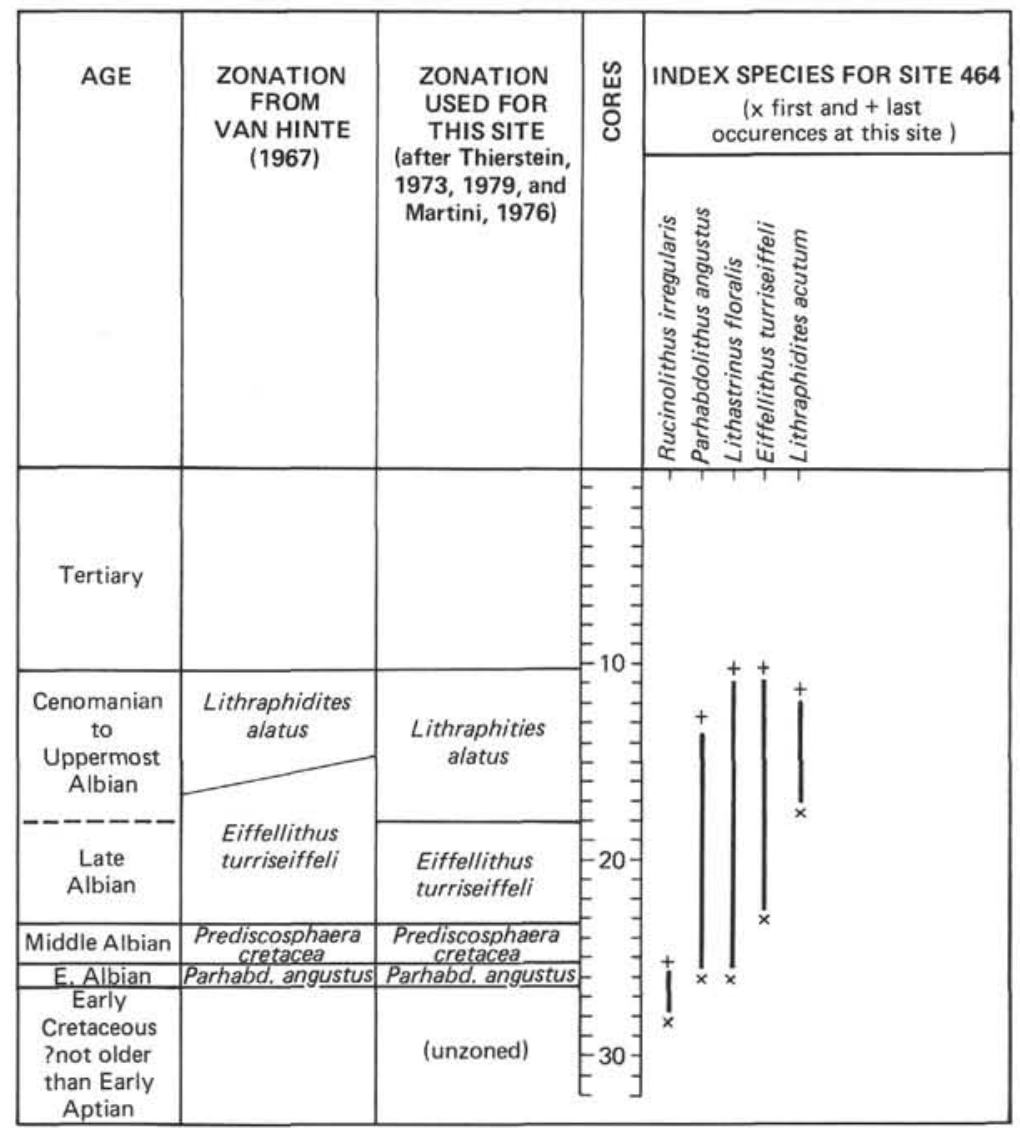

Figure 5. Ranges of index species at Site 464 and correlation of the zonation used with that according to van Hinte (1976).

Table 4. Distribution and abundance of calcareous-nannoplankton species in the Cretaceous sediments of Hole 465.

\begin{tabular}{|c|c|c|c|c|c|c|c|c|c|c|c|c|c|c|c|}
\hline $\begin{array}{c}\text { Sample } \\
\text { (interval } \\
\text { in } \mathrm{cm} \text { ) }\end{array}$ & $\begin{array}{l}\text { Sub- } \\
\text { bottom } \\
\text { Depth } \\
\text { (m) }\end{array}$ & 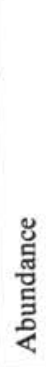 & 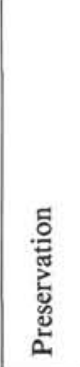 & $\stackrel{\circ}{<}$ & 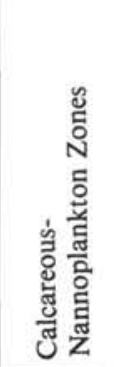 & 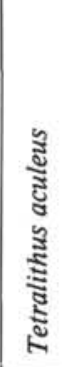 & 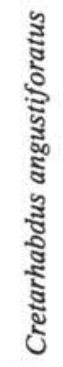 & 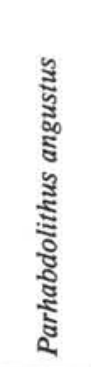 & 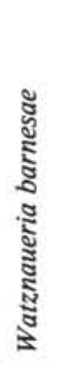 & 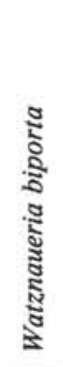 & 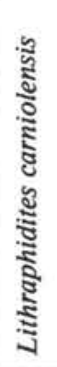 & 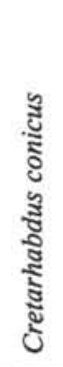 & 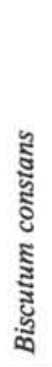 & 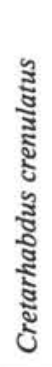 & 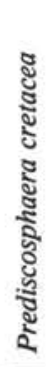 \\
\hline $465-9, \mathrm{CC}$ & 77.0 & A & M & & & $\mathrm{C}$ & VR & & A & VR & F & & & C & A \\
\hline $10-1,54-55$ & 77.54 & A & M & & & & $\mathrm{F}$ & & A & VR & $\mathrm{C}$ & & $\mathrm{C}$ & C & A \\
\hline $10-2,54-55$ & 79.04 & A & M & Late to & Lithra- & & C & C & A & & C & & & C & A \\
\hline $10-3,54-55$ & 80.54 & A & M & Middle & phidites & & $\mathrm{C}$ & $\mathrm{R}$ & A & VR & C & & $\mathrm{F}$ & $\mathrm{C}$ & A \\
\hline $10-4,54-55$ & 82.04 & A & M & Maast- & quad- & $\mathrm{F}$ & C & $\mathrm{F}$ & A & & C & & & C & A \\
\hline $10-5,54-55$ & 83.54 & A & $\mathrm{M}$ & richtian & ratus & C & C & $\mathrm{C}$ & A & $\mathrm{R}$ & F & & C & A & A \\
\hline $10, \mathrm{CC}$ & 86.50 & A & P-M & & & $\mathrm{F}$ & $\mathrm{R}$ & VR/X & A & & C & & & C & A \\
\hline $11, \mathrm{CC}$ & 96.0 & A & M & & & VR & $\mathrm{F}$ & C & A & & C & VR & $\mathrm{F}$ & $\mathrm{C}$ & A \\
\hline
\end{tabular}

Note: Symbols as in Table 2. 
blages of abundant nannofossils which contain Tetralithus trifidus (Stradner). The total range of this index species defines the Tetralithus trifidus Zone. In 465A$13, \mathrm{CC}$, there is a marked increase in etching and dissolution of the coccoliths.

Core $465 \mathrm{~A}-20$ belongs to the late Campanian Tetralithus gothicus Zone, as it contains the zone marker together with Arkhangelskiella cymbiformis Vekshina, Tetralithus aculeus (Stradner), and Broinsonia parca (Stradner), and abundant other nannoplankton species.

Lower to middle Campanian sediments were encountered from Samples 465A-21-3, 135-136 cm to 465A21-4, 43-44 cm; they contain Broinsonia parca (Stradner), Arkhangelskiella cymbiformis Vekshina, and Parhabdolithus regularis (Gorka), and indicate the Broinsonia parca Zone, as species of the genus Tetralithus are absent.

The abundant assemblages in Samples 465A-21,CC to $465 \mathrm{~A}-23, \mathrm{CC}$ are characterized by the lack of certain index species, such as Broinsonia parca (Stradner) and Marthasterites furcatus (Deflandre). The upper limit of this zonal interval is determined by the first occurrence of Broinsonia parca (Stradner) and Parhabdolithus regularis (Gorka), and the lower limit by the first occurrence of Effellithus eximius (Stover), Micula staurophora (Gardet), and Lithastrinus grilli Stradner, which has a known range from Coniacian to Santonian. It indicates that the age of the lower limit is not older than Coniacian.

The stratigraphic determination of Cores $465 \mathrm{~A}-24$ and $465 \mathrm{~A}-25$ is impossible, because only cherts and no fossiliferous sediments were recovered.

The top of the olive-gray laminated limestone (Samples $465 \mathrm{~A}-26-1,14-15 \mathrm{~cm}$ to $465 \mathrm{~A}-27, \mathrm{CC}$ ) contains abundant assemblages of coccoliths with the zone markers Lithraphidites alatus Thierstein and Lithra- phidites acutum Verbeek and Manivit, which assign this interval to the Lithraphidites alatus Zone (uppermost Albian to Cenomanian).

The oldest stratigraphic interval of Hole $465 \mathrm{~A}$ is characterized by nannofossil assemblages, which are meagre and generally poorly preserved. These coccolith assemblages contain the zone marker Eiffelithus turriseiffeli (Deflandre and Fert), but they lack Lithraphidites acutum Verbeek and Manivit and Lithraphidites alatus Thierstein (both index species of the Lithraphidites alatus Zone), and thus assign this interval to the Eiffellithus turriseiffeli Zone, of late Albian age.

\section{Site 466 \\ $\left(34^{\circ} 11.46^{\prime} \mathrm{N}, 179^{\circ} 15.34^{\prime} \mathrm{E}\right.$, depth $\left.2665 \mathrm{~m}\right)$}

Site 466 was drilled on southern Hess Rise, about 50 km northeast of Site 465 (Fig. 1). The total depth drilled in this hole was 312 meters. The Cretaceous sediments are represented by two lithologic units. The younger one, Upper Cretaceous cherty nannofossil ooze, includes the Tetralithus trifidus Zone, the Tetralithus gothicus Zone, the Broinsonia parca Zone, and an unzoned interval of middle Turonian to Santonian age. The older unit, with nannofossil chalk and limestone, and with pyritic clay, is late Albian to early Cenomanian in age (Eiffellithus turriseiffeli Zone). Zonation for Site 466 is shown in Figure 7. Distribution and abundance of species are shown in Table 6.

The abundant and moderately preserved nannofossils, including Tetralithus gothicus Deflandre, are found in Core 11 (Samples 11-1, 45-46 cm and 11,CC). The total range of this species defines the Tetralithus trifidus Zone, of late Campanian to early Maastrichtian age.

In Samples 12-1, 35-36 cm to $15, \mathrm{CC}$, predominantly common and moderately preserved late Campanian

Table 4. (Continued).

\begin{tabular}{|c|c|c|c|c|c|c|c|c|c|c|c|c|c|c|c|c|c|c|c|c|}
\hline 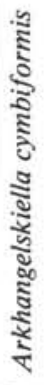 & 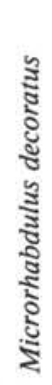 & 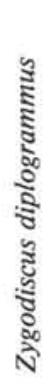 & 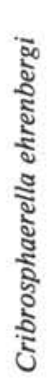 & 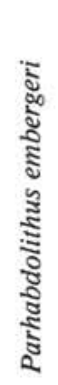 & 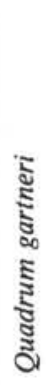 & 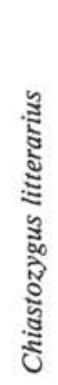 & 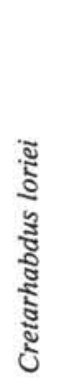 & 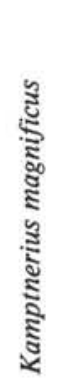 & 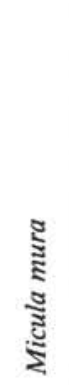 & 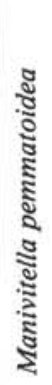 & 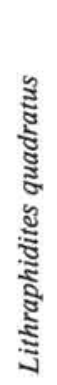 & 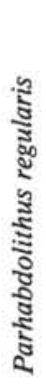 & 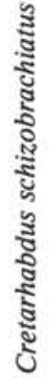 & 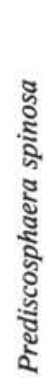 & 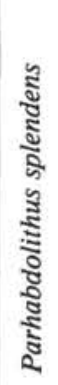 & 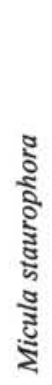 & 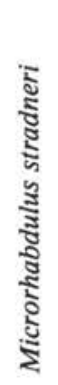 & 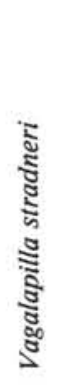 & 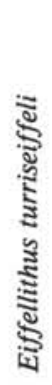 & 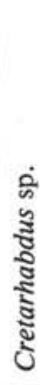 \\
\hline R & C & & A & R & $\mathrm{R}$ & C & VR & & $F / X$ & C & VR & C & & $R$ & & A & & & A & \\
\hline F & C & C & C & $\mathrm{R}$ & & $\mathrm{F}$ & & & & C & C & $\mathrm{F}$ & & & & C & C & $\mathrm{F}$ & A & C \\
\hline$F$ & C & C & A & VR & & C & & & & C & C & C & C & C & & C & C & C & C & \\
\hline$F$ & C & R & A & $\mathrm{F}$ & & A & VR & & & $R$ & C & C & C & $\mathrm{F}$ & $\mathrm{F}$ & C & C & C & C & \\
\hline \multirow[t]{2}{*}{ F } & C & F & A & C & & VR & $\mathrm{F}$ & VR & & $R$ & C & C & F & $\mathrm{R}$ & VR & C & VR & & C & \\
\hline & C & $F$ & A & F & & C & & & & F & F & C & C & C & $\mathrm{R}$ & C & C & & C & \\
\hline $\mathrm{F}$ & C & F & A & F & & $\mathrm{F}$ & & & & $\mathrm{F}$ & C & F & F & & $\mathrm{F}$ & C & C & $\mathrm{F}$ & C & \\
\hline C & C & & A & C & $\mathrm{F}$ & $R$ & & & & C & $\mathrm{F}$ & C & C & $\mathrm{F}$ & $R$ & A & C & & C & \\
\hline
\end{tabular}


Table 5. Distribution and abundance of calcareous-nannoplankton species in the Cretaceous sediments of Hole 465A.

\begin{tabular}{|c|c|c|c|c|c|c|c|c|c|c|c|c|c|c|c|c|c|}
\hline $\begin{array}{c}\text { Sample } \\
\text { (interval } \\
\text { in } \mathrm{cm} \text { ) }\end{array}$ & $\begin{array}{l}\text { Sub- } \\
\text { bottom } \\
\text { Depth } \\
\text { (m) }\end{array}$ & 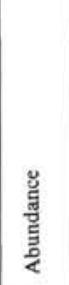 & 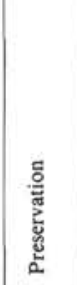 & \& & 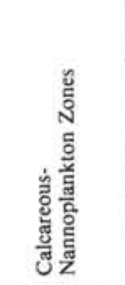 & 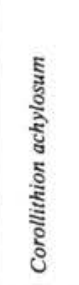 & 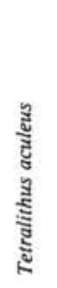 & 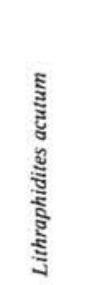 & 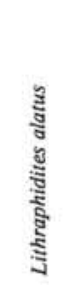 & 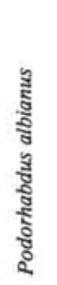 & 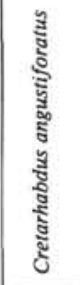 & 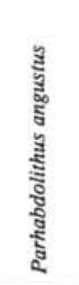 & 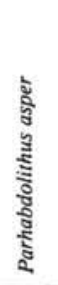 & 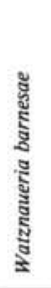 & 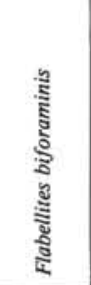 & 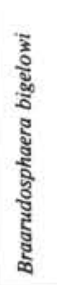 & 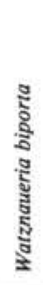 \\
\hline $\begin{array}{c}465 \mathrm{~A}-3-3,120 \\
3-4,2-3 \\
3, \mathrm{CC} \\
\end{array}$ & $\begin{array}{l}62.20 \\
62.52 \\
67.50 \\
\end{array}$ & $\begin{array}{l}\mathrm{A} \\
\mathrm{A} \\
\mathrm{A}\end{array}$ & $\begin{array}{c}\mathrm{M}-\mathrm{G} \\
\mathrm{M} \\
\mathrm{P}-\mathrm{M} \\
\end{array}$ & $\begin{array}{c}\text { Late } \\
\text { Maastr. }\end{array}$ & $\begin{array}{l}\text { Micula } \\
\text { mura }\end{array}$ & & VR & & & & $\begin{array}{l}\text { VR } \\
\mathrm{C} \\
\end{array}$ & $\begin{array}{l}F \\
F\end{array}$ & & $\begin{array}{l}\mathrm{A} \\
\mathrm{A} \\
\mathrm{A}\end{array}$ & & & $\begin{array}{c}\text { VR } \\
\text { F } \\
\text { VR } \\
\end{array}$ \\
\hline $4, \mathrm{CC}$ & 77.00 & $\mathrm{~A}$ & $\mathrm{P}$ & L.-M. Ma. & L. quad. & & $\mathbf{R}$ & & & & & & & C & & & VR \\
\hline $\begin{array}{l}6, \mathrm{CC} \\
8, \mathrm{CC} \\
9, \mathrm{CC} \\
10, \mathrm{CC} \\
11-4,41-42 \\
11-5,8-9 \\
\end{array}$ & $\begin{array}{r}96.00 \\
115.00 \\
124.50 \\
134.00 \\
138.91 \\
140.8 \\
\end{array}$ & $\begin{array}{l}\text { A } \\
\text { A } \\
\text { A } \\
\text { C } \\
\text { A } \\
\text { A }\end{array}$ & $\begin{array}{l}\mathrm{P}-\mathrm{M} \\
\mathrm{M} \\
\mathrm{M} \\
\mathrm{P} \\
\mathrm{M} \\
\mathrm{M} \\
\end{array}$ & $\begin{array}{c}\text { Early } \\
\text { Maast- } \\
\text { richtian }\end{array}$ & $\begin{array}{c}\text { Arkhangel- } \\
\text { skiella } \\
\text { cymbi- } \\
\text { formis }\end{array}$ & & $\begin{array}{l}\mathrm{C} \\
\mathrm{R} \\
\mathrm{R} \\
\mathrm{R} \\
\mathrm{R} \\
\mathrm{VR}\end{array}$ & & & & $\begin{array}{c}\mathrm{VR} / \mathrm{X} \\
\mathrm{F} / \mathrm{X} \\
\mathrm{VR} \\
\mathrm{R} \\
\end{array}$ & $\begin{array}{c}\mathrm{F} \\
\mathrm{F} \\
\mathrm{R} / \mathrm{X} \\
\mathrm{R} \\
\mathrm{VR} \\
\end{array}$ & & $\begin{array}{l}\text { A } \\
\text { A } \\
\text { A } \\
\text { C } \\
\text { A } \\
\text { A }\end{array}$ & & & $\begin{array}{c}\mathrm{F} \\
\mathrm{R} \\
\mathrm{VR}\end{array}$ \\
\hline $\begin{array}{l}11, \mathrm{CC} \\
12, \mathrm{CC} \\
13, \mathrm{CC} \\
14, \mathrm{CC} \\
15, \mathrm{CC} \\
16-2,30 \\
16, \mathrm{CC} \\
17, \mathrm{CC} \\
18, \mathrm{CC} \\
19, \mathrm{CC} \\
\end{array}$ & $\begin{array}{l}143.5 \\
153.0 \\
162.5 \\
172.0 \\
181.5 \\
183.30 \\
191.0 \\
200.5 \\
210.0 \\
219.5 \\
\end{array}$ & $\begin{array}{l}\mathrm{A} \\
\mathrm{A} \\
\mathrm{F} \\
\mathrm{A} \\
\mathrm{A} \\
\mathrm{A} \\
\mathrm{A} \\
\mathrm{A} \\
\mathrm{A} \\
\mathrm{A}\end{array}$ & $\begin{array}{c}M \\
P-M \\
P \\
P-M \\
M \\
M \\
M \\
P-M \\
P-M \\
M-G \\
M \\
\end{array}$ & $\begin{array}{l}\text { Early } \\
\text { Maast- } \\
\text { richtian } \\
\text { to Late } \\
\text { Campanian }\end{array}$ & $\begin{array}{l}\text { Tetralithus } \\
\text { trifidus }\end{array}$ & & $\begin{array}{l}\mathrm{F} \\
\mathrm{VR} \\
\mathrm{C} \\
\mathrm{C} \\
\\
\mathrm{F} \\
\mathrm{C} \\
\end{array}$ & & & & $\mathrm{R}$ & $\begin{array}{c}\mathrm{VR} \\
\mathrm{VR} / \mathrm{X}\end{array}$ & & $\begin{array}{l}\text { A } \\
\text { C } \\
\text { C } \\
\text { A } \\
\text { A } \\
\text { A } \\
\text { A } \\
\text { A } \\
\text { A } \\
\text { A }\end{array}$ & & & $\begin{array}{l}\mathrm{R} \\
\mathrm{R}\end{array}$ \\
\hline $\begin{array}{l}20-1,33-34 \\
20-2,33-34 \\
20-3,134-135 \\
20, C C \\
\end{array}$ & $\begin{array}{l}219.83 \\
221.33 \\
223.84 \\
229.00 \\
\end{array}$ & $\begin{array}{l}\text { A } \\
\text { A } \\
\text { A } \\
\text { A }\end{array}$ & $\begin{array}{c}M-G \\
M-G \\
M-G \\
M \\
\end{array}$ & $\begin{array}{c}\text { Late } \\
\text { Cam- } \\
\text { panian }\end{array}$ & $\begin{array}{c}\text { Tetra- } \\
\text { lithus } \\
\text { gothicus }\end{array}$ & & $\begin{array}{l}\mathrm{C} \\
\mathrm{C} \\
\mathrm{C} \\
\mathrm{C}\end{array}$ & & & & & & & $\begin{array}{l}\text { A } \\
\text { A } \\
\text { A } \\
\text { A }\end{array}$ & & & $\begin{array}{l}\text { VR } \\
\text { VR }\end{array}$ \\
\hline $\begin{array}{l}21-3,135-136 \\
21-4,43-44\end{array}$ & $\begin{array}{l}233.35 \\
233.93 \\
\end{array}$ & A & $\begin{array}{l}\mathrm{M} \\
\mathrm{M}\end{array}$ & $\begin{array}{l}\text { Mid. to Early } \\
\text { Campan. }\end{array}$ & $\begin{array}{l}\text { Broin. } \\
\text { parca }\end{array}$ & & & & & & & & & $\begin{array}{l}\mathrm{A} \\
\mathrm{A}\end{array}$ & & & VR \\
\hline $\begin{array}{l}21, \mathrm{CC} \\
22, \mathrm{CC} \\
23, \mathrm{CC}\end{array}$ & $\begin{array}{l}238.50 \\
248.0 \\
257.50\end{array}$ & ${ }^{\mathrm{A}}$ (ch & \begin{tabular}{|l} 
P \\
ert) \\
P-M
\end{tabular} & $\begin{array}{c}\text { Sant. } \\
\text { to } \\
\text { Coniac. }\end{array}$ & (unzoned) & & & & & & $\begin{array}{l}\text { VR } \\
\text { C }\end{array}$ & & & $\begin{array}{l}\mathrm{C} \\
\mathrm{A}\end{array}$ & & & F \\
\hline $\begin{array}{l}24, \mathrm{CC} \\
25, \mathrm{CC}\end{array}$ & $\begin{array}{l}267.0 \\
276.50\end{array}$ & & $\begin{array}{l}\text { ert) } \\
\text { ert) }\end{array}$ & & & & & & & & & & & & & & \\
\hline $\begin{array}{l}26-1,14-15 \\
26, \mathrm{CC} \\
27, \mathrm{CC} \\
\end{array}$ & $\begin{array}{l}276.64 \\
286.0 \\
295.50 \\
\end{array}$ & $\begin{array}{l}\mathrm{A} \\
\mathrm{R} \\
\mathrm{C} \\
\end{array}$ & \begin{tabular}{|c|}
$\mathrm{M}$ \\
$\mathrm{P}$ \\
$\mathrm{P}-\mathrm{M}$ \\
\end{tabular} & $\begin{array}{c}\text { Earliest } \\
\text { Turonian to } \\
\text { Latest Albian }\end{array}$ & $\begin{array}{l}\text { Lithrap. } \\
\text { alatus }\end{array}$ & VR & & VR & $\mathbf{F}$ & $\mathrm{F}$ & & $\begin{array}{l}\mathrm{C} \\
\mathrm{C}\end{array}$ & $\begin{array}{l}\mathrm{C} \\
\mathrm{F} \\
\mathrm{C}\end{array}$ & $\begin{array}{l}\mathrm{A} \\
\mathrm{C} \\
\mathrm{C}\end{array}$ & & & R \\
\hline $\begin{array}{l}28-1,58-59 \\
29-1,139 \\
30-1,24-25 \\
31-1,23-25 \\
32-1,60 \\
33, \mathrm{CC} \\
34, \mathrm{CC} \\
35, \mathrm{CC} \\
36, \mathrm{CC} \\
37-1,79-80 \\
38-2,39 \\
39-1,112 \\
40-1,139-140 \\
\end{array}$ & $\begin{array}{l}296.08 \\
306.39 \\
314.74 \\
324.23 \\
334.10 \\
352.50 \\
362.00 \\
371.50 \\
381.00 \\
381.79 \\
392.39 \\
401.12 \\
410.89 \\
\end{array}$ & $\begin{array}{l}\text { F-C } \\
\text { C-A } \\
\text { C-A } \\
\text { F-C } \\
\text { C } \\
\text { C-A } \\
\text { F-C } \\
\text { C-A } \\
\text { F } \\
\text { F } \\
\text { C } \\
\text { C } \\
\text { R } \\
\end{array}$ & $\begin{array}{c}\mathrm{P} \\
\mathrm{M} \\
\mathrm{P}-\mathrm{M} \\
\mathrm{P}-\mathrm{M} \\
\mathrm{P}-\mathrm{M} \\
\mathrm{M} \\
\mathrm{P}-\mathrm{M} \\
\mathrm{P}-\mathrm{M} \\
\mathrm{P} \\
\mathrm{P} \\
\mathrm{P}-\mathrm{M} \\
\mathrm{P}-\mathrm{M} \\
\mathrm{P} \\
\end{array}$ & $\begin{array}{c}\text { Late } \\
\text { Albian }\end{array}$ & $\begin{array}{l}\text { Eiffel- } \\
\text { lithus } \\
\text { turris- } \\
\text { eiffeli }\end{array}$ & $\begin{array}{c}R \\
R \\
V R / X \\
V R / X\end{array}$ & & $\mathrm{VR} / \mathrm{X}$ & VR/X & $\begin{array}{c}\text { VR } \\
\text { F } \\
\text { VR } \\
\text { F } \\
\text { F } \\
F \\
\text { R }\end{array}$ & & $\begin{array}{l}\mathrm{F} \\
\mathrm{F} \\
\mathrm{F} \\
\mathrm{F} \\
\mathrm{F} \\
\mathrm{F} \\
\mathrm{F} \\
\mathrm{F} \\
\mathrm{R} \\
\mathrm{F} \\
\mathrm{F} \\
\mathrm{F} \\
\mathrm{VR} \\
\end{array}$ & $\begin{array}{l}\text { F } \\
\text { C } \\
\text { C } \\
\text { C } \\
\text { F } \\
\text { C } \\
\text { F } \\
\text { C } \\
\text { F } \\
\text { C } \\
\text { C } \\
\text { C } \\
\text { VR } \\
\end{array}$ & $\begin{array}{l}\text { C } \\
\text { C } \\
\text { A } \\
\text { C } \\
\text { C } \\
\text { C } \\
\text { A } \\
\text { A } \\
\text { C } \\
\text { C } \\
\text { C } \\
\text { A } \\
\text { A }\end{array}$ & $\begin{array}{c}\mathrm{VR} / \mathrm{X} \\
\mathrm{VR} \\
\end{array}$ & $\begin{array}{l}\mathrm{F} \\
\mathrm{C}\end{array}$ & $\mathbf{R}$ \\
\hline $\begin{array}{l}40-2,39 \\
40-2,65-66\end{array}$ & $\begin{array}{l}411.39 \\
411.65\end{array}$ & \multicolumn{2}{|c|}{$\begin{array}{l}\text { (barren) } \\
\text { (barren) }\end{array}$} & $?$ & $?$ & & & & & & & & & & & & \\
\hline
\end{tabular}

Note: Symbols as in Table 2.

assemblages occur with the zone marker Tetralithus gothicus Deflandre, indicating the Tetralithus gothicus Zone, as Tetralithus trifidus (Stradner) is absent.

The thickness of the Broinsonia parca Zone is only a few meters (Samples 16-1, 55-56 cm to $16-3,55-56 \mathrm{~cm}$ ). Besides the zone marker Broinsonia parca (Stradner), Parhabdolithus regularis (Gorka) and Arkhangelskiella cymbiformis Vekshina are present, but Tetralithus gothicus Deflandre is missing.

The interval between Samples $16, \mathrm{CC}$ to $23, \mathrm{CC}$ is characterized by the lack of Broinsonia parca (Stradner) and Marthasterites furcatus (Deflandre). The upper part of this interval (Cores 16-18) contains assemblages of abundant coccoliths; in the lower part (Cores 19-23), the nannofossils are few to common. The upper limit of this stratigraphic interval (late Turonian to Santonian) is determined by the first occurrence of Broinsonia parca (Stradner) and Arkhangelskiella cymbiformis Vekshina, and the lower limit by the first occurrence of Eiffellithus eximius (Stover) and Micula staurophora (Gardet).

No stratigraphic evidence was found in Cores 24 to 28; samples were either barren or not available. Samples from the late Albian interval (Samples 29-1, 34-36 cm to $35, \mathrm{CC}$ ) contain predominantly poorly preserved coccoliths, including Eiffellithus turriseiffeli (Deflandre and Fert). The assemblages lack Lithraphidites alatus Thierstein, an index species of Cenomanian age, and are thus 
Table 5. (Continued).

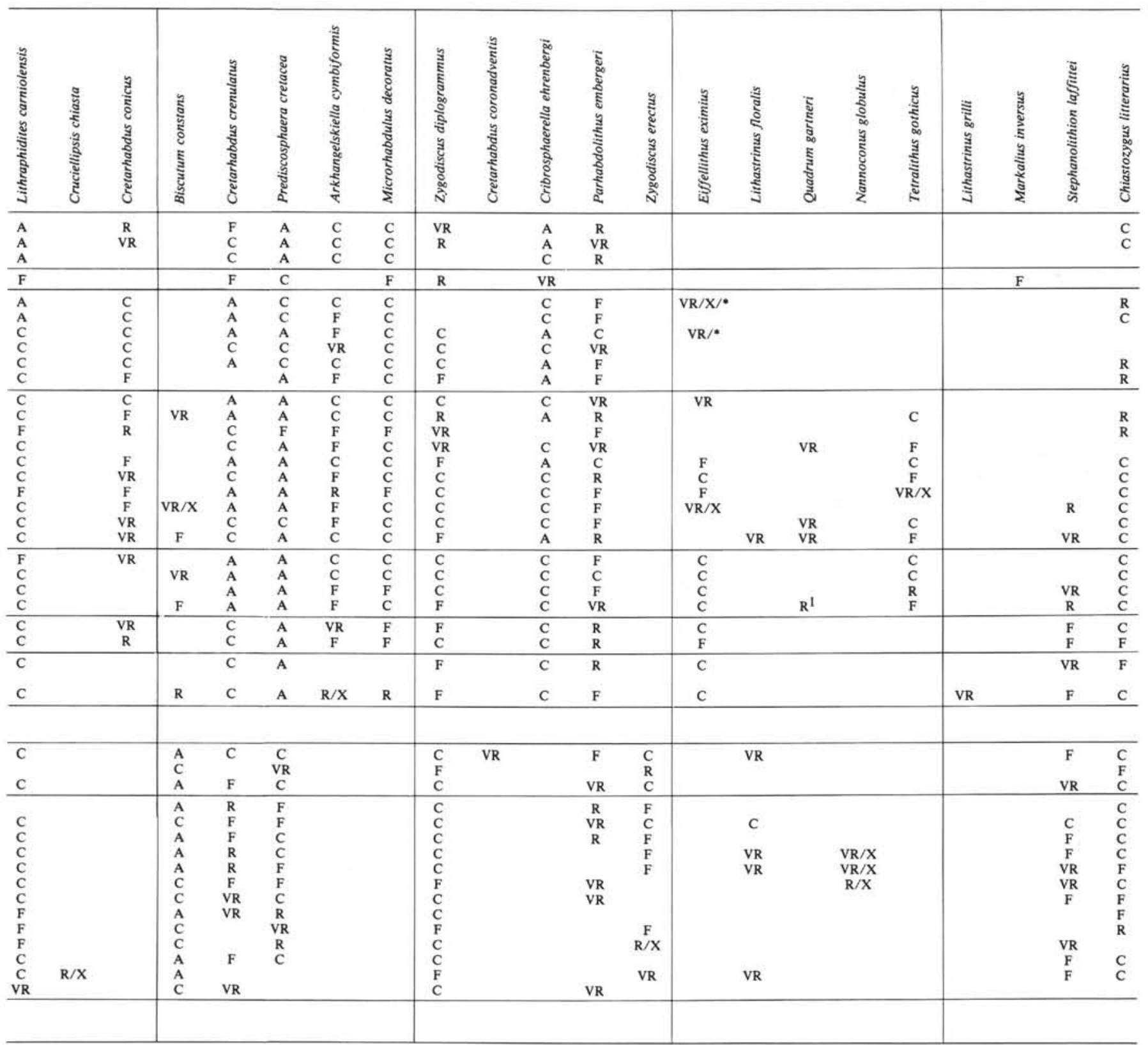

assigned to the Eiffellithus turriseiffeli Zone of late Albian age.

\section{ACKNOWLEDGMENTS}

Primary support was provided by the Deutsche Forschungsgemeinschaft, Bad Godesberg, and the Bundesanstalt für Geowissenschaften und Rohstoffe, Hannover. Core materials were made available by the Deep Sea Drilling Project. I with to thank Erlend Martini (Johann-Wolfgang-Goethe-Universität, Frankfurt), Thierry Moorkens (Texaco AG., Wietze), and Herbert Stradner (Geologische Bundesanstalt, Wien) for reviewing an early draft of the manuscript.

\section{REFERENCES}

Bukry, D., 1974. Coccolith stratigraphy, offshore Western Australia, Deep Sea Drilling Project Leg 27. In Veevers, J. J., Heirtzler, J. R., et al., Init. Repts. DSDP, 27: Washington (U.S. Govt. Printing Office), 623-630.
Bukry, D., and Bramlette, M. N., 1970. Coccolith age determinations Leg 3, Deep Sea Drilling Project. In Maxwell, A. E., von Herzen, R., et al., Init. Repts. DSDP, 3: Washington (U.S. Govt. Printing Office), 589-611.

Čepek, P., 1978. Mesozoic calcareous nannoplankton of the eastern North Atlantic, Leg 41. In Lancelot, Y., Seibold, E., et al., Init. Repts. DSDP, 41: Washington (U.S. Govt. Printing Office), 667-687.

Čepek, P., and Hay, W. W., 1969. Calcareous nannoplankton and biostratigraphic subdivision of the Upper Cretaceous. Gulf Coast Assoc. Geol. Soc. Trans., 19:323-336.

Gartner, S., 1972. Late Pleistocene calcareous nannofossils in the Caribbean and their interoceanic correlation. Palaeogeography, Palaeoclimatology, Palaeoecology, 12:169-191.

Hay, W. W, 1970. Calcareous nannofossils from cores recovered on Leg 4. In Bader, R. G., Gerard, R. D., et al., Init. Repts. DSDP, 4: Washington (U.S. Govt. Printing Office), 455-503. 


\section{P. ČEPEK}

Table 5. (Continued),

\begin{tabular}{|c|c|c|c|c|c|c|c|c|c|c|c|c|c|c|c|c|c|}
\hline $\begin{array}{c}\text { Sample } \\
\text { (interval } \\
\text { in } \mathrm{cm} \text { ) }\end{array}$ & $\begin{array}{l}\text { Sub- } \\
\text { bottom } \\
\text { Depth } \\
\text { (m) }\end{array}$ & $\begin{array}{l}\text { : } \\
\text { हूँ } \\
\text { है } \\
\text { \& }\end{array}$ & 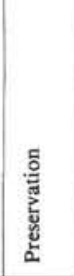 & 橤 & 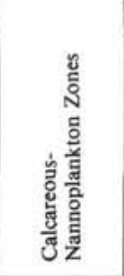 & 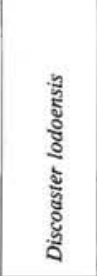 & 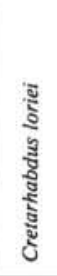 & 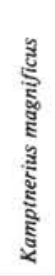 & 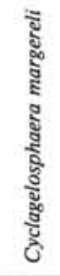 & 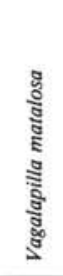 & 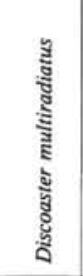 & 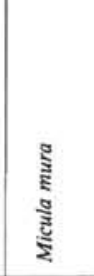 & 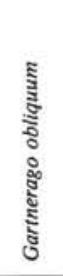 & 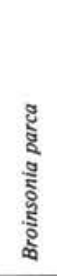 & 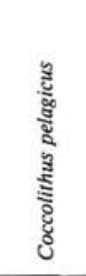 & 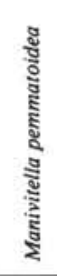 & 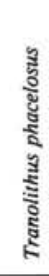 \\
\hline $\begin{array}{c}465 \mathrm{~A}-3-3,120 \\
3-4,2-3 \\
3, \mathrm{CC} \\
\end{array}$ & $\begin{array}{l}62.20 \\
62.52 \\
67.50 \\
\end{array}$ & $\begin{array}{l}\mathrm{A} \\
\mathrm{A} \\
\mathrm{A}\end{array}$ & $\begin{array}{c}\mathrm{M}-\mathrm{G} \\
\mathrm{M} \\
\mathrm{P}-\mathrm{M} \\
\end{array}$ & $\begin{array}{c}\text { Late } \\
\text { Maastr. }\end{array}$ & $\begin{array}{c}\text { Micula } \\
\text { mura }\end{array}$ & & & & & & & $\begin{array}{l}\mathrm{C} \\
\mathrm{F} \\
\mathrm{C}\end{array}$ & & & & $\begin{array}{l}\mathrm{C} \\
\mathrm{F} \\
\mathrm{C}\end{array}$ & \\
\hline $4, \mathrm{CC}$ & 77.00 & A & $\mathrm{P}$ & L. $-\overline{\mathrm{M}} \cdot \overline{\mathrm{Ma}}$ & L. quad. & $\mathrm{VR} /(+)$ & & & & & $\operatorname{VR}(+)$ & & & & $\mathrm{C} /(+)$ & $F$ & 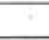 \\
\hline $\begin{array}{l}6, \mathrm{CC} \\
8, \mathrm{CC} \\
9, \mathrm{CC} \\
10, \mathrm{CC} \\
11-4,41-42 \\
11-5,8-9\end{array}$ & $\begin{array}{r}96.00 \\
115.00 \\
124.50 \\
134.00 \\
138.91 \\
140.8\end{array}$ & $\begin{array}{l}\text { A } \\
\text { A } \\
\text { A } \\
\text { C } \\
\text { A } \\
\text { A }\end{array}$ & $\begin{array}{l}\mathrm{P}-\mathrm{M} \\
\mathrm{M} \\
\mathrm{M} \\
\mathrm{P} \\
\mathrm{M} \\
\mathrm{M}\end{array}$ & $\begin{array}{c}\text { Early } \\
\text { Maast- } \\
\text { richtian }\end{array}$ & $\begin{array}{c}\text { Arkhangel- } \\
\text { skiella } \\
\text { cymbi- } \\
\text { formis }\end{array}$ & & $\begin{array}{l}\text { VR } \\
\text { VR } \\
\text { VR }\end{array}$ & & & & & & & & & $\begin{array}{l}\text { C } \\
\text { C } \\
\text { C } \\
\text { F } \\
\text { C } \\
\text { C }\end{array}$ & VR \\
\hline $\begin{array}{l}11, \mathrm{CC} \\
12, \mathrm{CC} \\
13, \mathrm{CC} \\
14, \mathrm{CC} \\
15, \mathrm{CC} \\
16-2,30 \\
16, \mathrm{CC} \\
17, \mathrm{CC} \\
18, \mathrm{CC} \\
19, \mathrm{CC} \\
\end{array}$ & $\begin{array}{l}143.5 \\
153.0 \\
162.5 \\
172.0 \\
181.5 \\
183.30 \\
191.0 \\
200.5 \\
210.0 \\
219.5 \\
\end{array}$ & $\begin{array}{l}\text { A } \\
\text { A } \\
\text { F } \\
\text { A } \\
\text { A } \\
\text { A } \\
\text { A } \\
\text { A } \\
\text { A } \\
\text { A }\end{array}$ & $\begin{array}{c}M \\
P-M \\
P \\
P-M \\
M \\
M \\
P-M \\
P-M \\
M-G \\
M \\
\end{array}$ & $\begin{array}{c}\text { Early } \\
\text { Maast- } \\
\text { richtian } \\
\text { to Late } \\
\text { Campanian }\end{array}$ & $\begin{array}{l}\text { Tetralithus } \\
\text { trifidus }\end{array}$ & & $\begin{array}{l}\mathrm{R} \\
\mathrm{R}\end{array}$ & & VR & & & VR(+) & VR & $\begin{array}{c}\text { VR } \\
\\
\text { C } \\
\text { C } \\
\text { F } \\
\text { VR } \\
\text { F } \\
\text { R }\end{array}$ & & $\begin{array}{l}C \\
C \\
\text { VR } \\
\text { R } \\
\text { C } \\
C \\
C \\
C \\
C \\
C \\
C \\
\end{array}$ & $\begin{array}{l}\text { VR } \\
\text { VR } \\
\\
\text { F } \\
\text { VR } \\
\text { VR } \\
\text { R } \\
\text { F } \\
\text { VR }\end{array}$ \\
\hline $\begin{array}{l}20-1,33-34 \\
20-2,33-34 \\
20-3,134-135 \\
20, \mathrm{CC}\end{array}$ & $\begin{array}{l}219.83 \\
221.33 \\
223.84 \\
229.00\end{array}$ & $\begin{array}{l}\text { A } \\
\text { A } \\
\text { A } \\
\text { A }\end{array}$ & $\begin{array}{c}M-G \\
M-G \\
M-G \\
M\end{array}$ & $\begin{array}{l}\text { Late } \\
\text { Cam- } \\
\text { panian }\end{array}$ & $\begin{array}{c}\text { Tetra- } \\
\text { lithus } \\
\text { gothicus }\end{array}$ & & $\begin{array}{l}\mathrm{F} \\
\mathrm{R}\end{array}$ & $\begin{array}{c}\text { VR } \\
\text { R }\end{array}$ & $\begin{array}{l}\mathrm{R} \\
\mathrm{VR}\end{array}$ & & & & $\begin{array}{l}\text { VR } \\
\text { R } \\
\text { VR }\end{array}$ & $\begin{array}{l}\mathrm{F} \\
\mathrm{F} \\
\mathrm{F} \\
\mathrm{VR}\end{array}$ & & $\begin{array}{l}\mathrm{C} \\
\mathrm{C} \\
\mathrm{C} \\
\mathrm{C}\end{array}$ & $\begin{array}{c}\mathrm{R} \\
\text { VR } \\
\mathrm{F} \\
\mathrm{R} \\
\end{array}$ \\
\hline $\begin{array}{l}21-3,135-136 \\
21-4,43-44\end{array}$ & $\begin{array}{l}233.35 \\
233.93\end{array}$ & $\begin{array}{l}\mathrm{A} \\
\mathrm{A}\end{array}$ & $\begin{array}{l}\mathrm{M} \\
\mathrm{M}\end{array}$ & $\begin{array}{l}\text { Mid. to Early } \\
\text { Campan. }\end{array}$ & $\begin{array}{l}\text { Broin. } \\
\text { parca }\end{array}$ & & & & & & & & VR & $\begin{array}{c}\text { VR } \\
\mathrm{R}\end{array}$ & & $\begin{array}{l}\mathrm{C} \\
\mathrm{C}\end{array}$ & $\begin{array}{l}\mathbf{R} \\
\mathbf{R} \\
\end{array}$ \\
\hline $\begin{array}{l}21, \mathrm{CC} \\
22, \mathrm{CC} \\
23, \mathrm{CC}\end{array}$ & $\begin{array}{l}238.50 \\
248.0 \\
257.50\end{array}$ & \multicolumn{2}{|c|}{\begin{tabular}{l|l} 
A & \\
\multicolumn{2}{c}{ (chert) } \\
A P-M
\end{tabular}} & $\begin{array}{c}\text { Sant. } \\
\text { to } \\
\text { Coniac. }\end{array}$ & (unzoned) & & & & VR & & & & & & & F & VR \\
\hline $\begin{array}{l}23, \mathrm{CC} \\
24, \mathrm{CC} \\
25, \mathrm{CC}\end{array}$ & $\begin{array}{l}257.50 \\
267.0 \\
276.50\end{array}$ & \multicolumn{2}{|c|}{$\begin{array}{l}\text { (chert) } \\
\text { (chert) }\end{array}$} & Coniac. & & & & & & & & & $\mathbf{R}$ & & & $\mathbf{F}$ & \\
\hline $\begin{array}{l}26-1,14-15 \\
26, C C \\
27, \mathrm{CC}\end{array}$ & $\begin{array}{l}276.64 \\
286.0 \\
295.50 \\
\end{array}$ & $\begin{array}{l}\mathrm{A} \\
\mathrm{R} \\
\mathrm{C} \\
\end{array}$ & \begin{tabular}{|c|}
$\mathrm{M}$ \\
$\mathrm{P}$ \\
$\mathrm{P}-\mathrm{M}$ \\
\end{tabular} & $\begin{array}{c}\text { Earliest } \\
\text { Turonian to } \\
\text { Latest Albian }\end{array}$ & $\begin{array}{c}\text { Lithrap. } \\
\text { alatus }\end{array}$ & & & & & $\mathrm{R}$ & & & & & & $\begin{array}{c}\mathrm{C} \\
\text { VR } \\
\mathrm{F}\end{array}$ & $\begin{array}{l}\mathrm{F} \\
\mathrm{C} \\
\mathrm{C}\end{array}$ \\
\hline $\begin{array}{l}28-1,58-59 \\
29-1,139 \\
30-1,24-25 \\
31-1,23-25 \\
32-1,60 \\
33, C C \\
34, C C \\
35, \mathrm{CC} \\
36, \mathrm{CC} \\
37-1,79-80 \\
38-2,39 \\
39-1,112 \\
40-1,139-140\end{array}$ & $\begin{array}{l}296.08 \\
306.39 \\
314.74 \\
324.23 \\
334.10 \\
352.50 \\
362.00 \\
371.50 \\
381.00 \\
381.79 \\
392.39 \\
401.12 \\
410.89 \\
\end{array}$ & $\begin{array}{l}\text { F-C } \\
\text { C-A } \\
\text { C-A } \\
\text { F-C } \\
\text { C } \\
\text { C-A } \\
\text { F-C } \\
\text { C-A } \\
\text { F } \\
\text { F } \\
\text { C } \\
\text { C } \\
\text { R }\end{array}$ & \begin{tabular}{|c}
$P$ \\
$M$ \\
$P-M$ \\
$P-M$ \\
$P-M$ \\
$M$ \\
$P-M$ \\
$P-M$ \\
$P$ \\
$P$ \\
$P-M$ \\
$P-M$ \\
$P$
\end{tabular} & $\begin{array}{c}\text { Late } \\
\text { Albian }\end{array}$ & $\begin{array}{l}\text { Eiffel- } \\
\text { lithus } \\
\text { turris- } \\
\text { eiffeli }\end{array}$ & & & & & VR & $\operatorname{VR}(+)$ & & & & & $\begin{array}{l}\text { F } \\
\text { F } \\
\text { F } \\
\text { F } \\
\text { C } \\
\text { F } \\
\text { VR } \\
\text { VR } \\
\text { VR } \\
\text { VR } \\
\text { R } \\
\text { C } \\
\text { F }\end{array}$ & $\begin{array}{c}\mathrm{F} \\
\text { VR } \\
\mathrm{F} \\
\mathrm{F} \\
\mathrm{F} \\
\mathrm{C} \\
\mathrm{F} \\
\\
\mathrm{R} \\
\mathrm{F} \\
\mathrm{R}\end{array}$ \\
\hline $\begin{array}{l}40-2,39 \\
40-2,65-66\end{array}$ & $\begin{array}{l}411.39 \\
411.65\end{array}$ & \multicolumn{2}{|c|}{$\begin{array}{l}\text { (barren) } \\
\text { (barren) }\end{array}$} & $?$ & $?$ & & & & & & & & & & & & \\
\hline
\end{tabular}

Note: Symbols as in Table 2.

Manivit, H., 1971. Les nannofossiles du Crétace francais de l'Aptien au Danien, essai de biozonation appuyee sur les stratitypes [Thése.] Fac. Science d'Orsay, France.

Martini, E., 1976. Cretaceous to Recent nannoplankton from the central Pacific Ocean (DSDP Leg 33). In Schlanger, S. O., Jackson, E. D., et al., Init. Repts. DSDP, 33: Washington (U.S. Govt. Printing Office), 383-424.

Perch-Nielsen, K., 1972. Remarks on the Late Cretaceous to Pleistocene coccoliths from the North Atlantic. In Laughton, A. S., Berggren, W. A., et al., Init. Repts. DSDP, 12: Washington (U.S. Govt. Printing Office), 1003-1069.

Roth, P. H., 1973. Calcareous nannofossils Leg 17 Deep Sea Drilling Project. In Winterer, E. L., Ewing, J. I., et al., Init. Repts. DSDP, 17: Washington (U.S. Govt. Printing Office), 695-796.
Thierstein, H. R., 1971. Tentative Lower Cretaceous calcareous nannoplankton zonation. Eclog. Geol. Helv., 64:459-488.

,1973. Lower Cretaceous calcareous nannoplankton biostratigraphy. Abh. Geol. B.-A., Wien, 29.

1976. Mesozoic calcareous nannoplankton biostratigraphy of marine sediments. Mar. Micropaleont., 1:325-362.

van Hinte, J. E., 1976. A Cretaceous time scale. Bull. Am. Assoc. Petrol. Geol., 60:498-516.

Verbeek, J. W., 1976. Upper Cretaceous calcareous nannoplankton from Ballon and Theligny, in the type area of the Cenomanian Stage (Sarthe, France). Proc. Koninkl. Nederl. Akad. Wetensch., 79:69-82.

1977. Calcareous nannoplankton biostratigraphy of middle and Upper Cretaceous deposits in Tunisia, Southern Spain and France. Utrecht Micropaleont. Bull., 16:1-157. 
Table 5. (Continued).

\begin{tabular}{|c|c|c|c|c|c|c|c|c|c|c|c|c|c|c|c|c|c|c|c|c|}
\hline 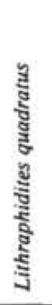 & 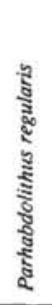 & 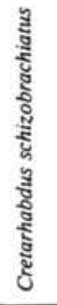 & 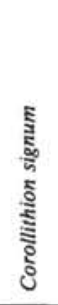 & 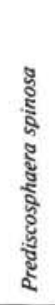 & 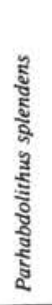 & 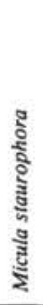 & 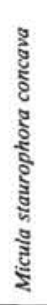 & 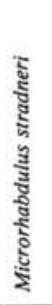 & 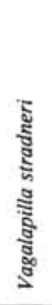 & $\begin{array}{l}\text { हैँ } \\
\text { हूँ } \\
\text { हूँ } \\
\text { हूँ } \\
\text { है }\end{array}$ & 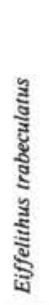 & 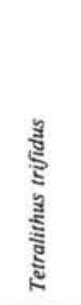 & 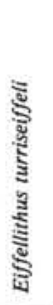 & 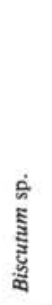 & 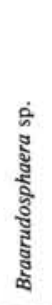 & $\begin{array}{l}\text { के } \\
\text { है } \\
\text { हैँ } \\
\text { हूँ } \\
\text { है }\end{array}$ & 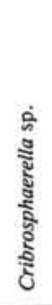 & 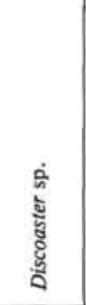 & 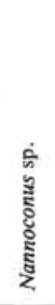 & 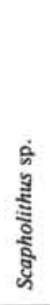 \\
\hline $\begin{array}{c}\text { C } \\
\text { F } \\
\text { VR }\end{array}$ & $\begin{array}{l}\mathrm{C} \\
\mathrm{C} \\
\mathrm{R}\end{array}$ & $\begin{array}{l}\mathrm{F} \\
\mathrm{F}\end{array}$ & & & & $\begin{array}{l}\text { A } \\
\text { A } \\
\text { C }\end{array}$ & C & & VR & & & & $\begin{array}{l}\mathrm{C} \\
\mathrm{C} \\
\mathrm{C}\end{array}$ & & & & & & & \\
\hline $\mathrm{R}$ & & & & & & $\mathrm{F}$ & & & & $F /(+)$ & & $\mathrm{R} /{ }^{*}$ & $\mathrm{~F}$ & & & & & $\operatorname{VR}(+)$ & & \\
\hline & $\begin{array}{c}\mathrm{F} \\
\mathrm{R} \\
\mathrm{F} \\
\mathrm{V} R\end{array}$ & $\begin{array}{c}\mathrm{R} \\
\mathrm{F} \\
\mathrm{VR} \\
\mathrm{VR} \\
\mathrm{R} \\
\mathrm{VR} \\
\end{array}$ & & $\begin{array}{l}\text { VR } \\
\text { VR }\end{array}$ & $\begin{array}{c}\text { VR } \\
\mathrm{F}\end{array}$ & $\begin{array}{l}\text { C } \\
\text { C } \\
\text { C } \\
\text { R } \\
\text { F } \\
\text { A }\end{array}$ & & $\begin{array}{l}\text { VR } \\
\text { VR }\end{array}$ & $\begin{array}{c}\text { VR } \\
\text { F } \\
\text { VR }\end{array}$ & & & & $\begin{array}{l}\mathrm{C} \\
\mathrm{C} \\
\mathrm{C} \\
\mathrm{C} \\
\mathrm{C} \\
\mathrm{C}\end{array}$ & & & & & & & \\
\hline & $R$ & $\mathbf{F}$ & & VR & & $\begin{array}{l}\mathrm{C} \\
\mathrm{F} \\
\mathrm{F} \\
\mathrm{F} \\
\mathrm{F} \\
\mathrm{F} \\
\mathrm{F} \\
\end{array}$ & VR & VR & VR & $\operatorname{VR}(+)$ & $R$ & $\begin{array}{c}\text { VR } \\
C \\
\text { F } \\
\text { VR/X } \\
C \\
\text { R } \\
\\
\text { C } \\
F \\
\end{array}$ & $\begin{array}{l}\mathrm{C} \\
\mathrm{C} \\
\mathrm{R} \\
\mathrm{C} \\
\mathrm{C} \\
\mathrm{C} \\
\mathrm{C} \\
\mathrm{C} \\
\mathrm{C} \\
\mathrm{C}\end{array}$ & & & & & & & \\
\hline & VR & & & $\begin{array}{l}\mathrm{R} \\
\mathrm{C}\end{array}$ & & $\begin{array}{l}\text { C } \\
\text { C } \\
\text { C } \\
F \\
\end{array}$ & $\begin{array}{l}\mathbf{R} \\
\mathbf{R} \\
\end{array}$ & & VR & & & & $\begin{array}{l}\text { C } \\
\text { C } \\
\text { C } \\
\text { F }\end{array}$ & & & & & & & \\
\hline & $\begin{array}{l}\mathbf{R} \\
\mathbf{R} \\
\end{array}$ & & & VR & & $\begin{array}{l}\text { c } \\
\text { c }\end{array}$ & & & & & & & $\begin{array}{l}\mathrm{C} \\
\mathrm{C}\end{array}$ & & VR & & & & & \\
\hline & & & & & & $\begin{array}{l}\mathbf{R} \\
\mathrm{C}\end{array}$ & & & & & c & & $\begin{array}{l}\text { VR } \\
\text { F }\end{array}$ & & & & & & & \\
\hline & & & $\begin{array}{c}\text { VR } \\
\text { C }\end{array}$ & & & & & & $\begin{array}{l}\mathrm{C} \\
\mathrm{R} \\
\mathrm{R}\end{array}$ & & $\begin{array}{l}\text { VR } \\
\text { C }\end{array}$ & & $\begin{array}{l}\mathrm{A} \\
\mathrm{F} \\
\mathrm{A}\end{array}$ & & & & $R$ & & VR & F \\
\hline & & & $\begin{array}{c}\text { VR } \\
\text { F } \\
\text { VR } \\
\text { R }\end{array}$ & & & & & & $\begin{array}{c}\mathrm{F} \\
\mathrm{F} \\
\mathrm{F} \\
\mathrm{C} \\
\mathrm{F} \\
\mathrm{F} \\
\mathrm{VR} \\
\mathrm{VR} \\
\mathrm{F} \\
\mathrm{C} \\
\mathrm{C} \\
\mathrm{F}\end{array}$ & & $F$ & & $\begin{array}{l}\text { C } \\
\text { C } \\
\text { C } \\
\text { C } \\
\text { C } \\
\text { C } \\
\text { C } \\
\text { C } \\
\text { C } \\
\text { C } \\
\text { A } \\
\text { C } \\
\mathrm{F}\end{array}$ & F & & . & $\begin{array}{c}\mathrm{R} \\
\mathrm{VR} \\
\mathrm{VR} \\
\mathrm{R}\end{array}$ & & $\begin{array}{c}\text { R } \\
F \\
\text { VR } \\
\text { F } \\
\text { VR } \\
\text { F } \\
\text { C } \\
\text { C }\end{array}$ & $\begin{array}{c}\text { VR } \\
\text { F }\end{array}$ \\
\hline
\end{tabular}


P. ČEPEK

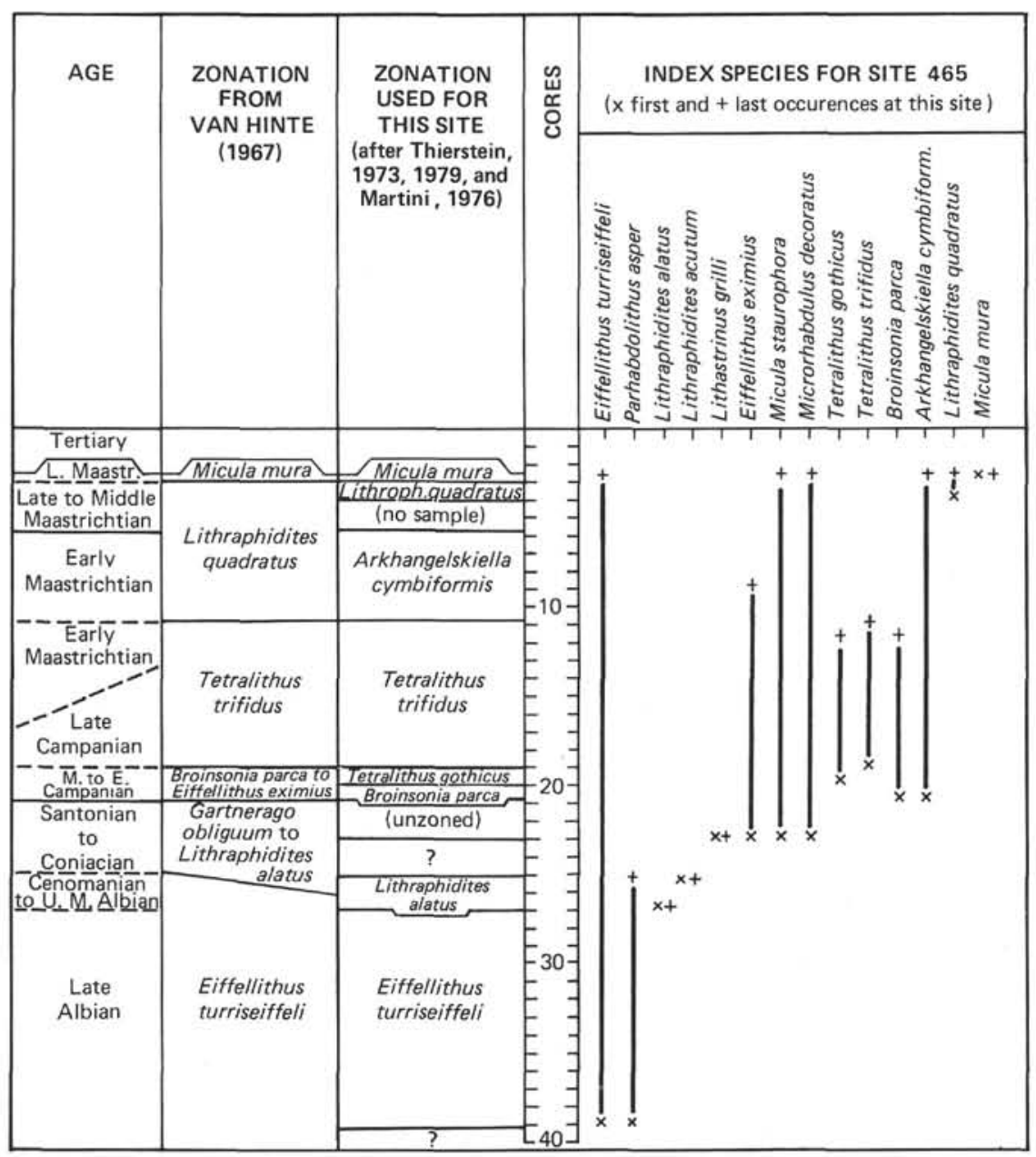

Figure 6. Ranges of index species at Site 465 and correlation of the zonation used with that according to van Hinte (1976). 

P. ČEPEK

Table 6. Distribution and abundance of calcareous-nannoplankton species in the Cretaceous sediments of Site 466.

\begin{tabular}{|c|c|c|c|c|c|c|c|c|c|c|c|c|c|c|c|}
\hline $\begin{array}{c}\text { Sample } \\
\text { (interval } \\
\text { in } \mathrm{cm} \text { ) }\end{array}$ & $\begin{array}{l}\text { Sub- } \\
\text { bottom } \\
\text { Depth } \\
\text { (m) }\end{array}$ & & & & & 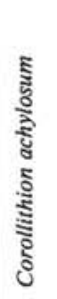 & 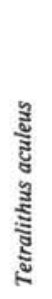 & 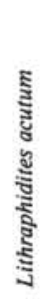 & 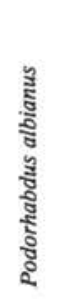 & 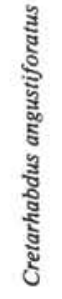 & 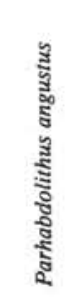 & 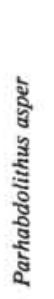 & 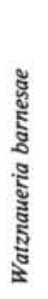 & 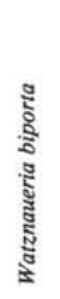 & 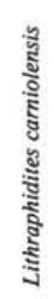 \\
\hline $\begin{array}{c}466-11-1,45-46 \\
11, C C\end{array}$ & $\begin{array}{l}88.45 \\
93.50 \\
\end{array}$ & $\begin{array}{l}\mathrm{A} \\
\mathrm{A}\end{array}$ & $\begin{array}{l}\mathrm{M} \\
\mathrm{M}\end{array}$ & $\begin{array}{c}\text { E. Maa. } \\
\text { to L. Cam. }\end{array}$ & $\begin{array}{l}\text { Tetral. } \\
\text { trifidius }\end{array}$ & & $\begin{array}{l}\mathrm{C} \\
\mathrm{R}\end{array}$ & & & & $\begin{array}{c}\text { VR } \\
\text { F }\end{array}$ & & $\begin{array}{l}\text { A } \\
\text { A }\end{array}$ & & $\begin{array}{l}\mathrm{C} \\
\mathrm{F}\end{array}$ \\
\hline $\begin{array}{l}12-1,35-36 \\
12, \mathrm{CC} \\
13-1,40-41 \\
13-3,35-37 \\
13-5,84-85 \\
13, \mathrm{CC} \\
14, \mathrm{CC} \\
15-1,42-43 \\
15-4,42-43 \\
15, \mathrm{CC} \\
\end{array}$ & $\begin{array}{r}93.85 \\
103.00 \\
103.40 \\
106.35 \\
109.84 \\
112.50 \\
122.00 \\
122.42 \\
126.92 \\
131.50 \\
\end{array}$ & $\begin{array}{l}\text { A } \\
\text { A } \\
\text { A } \\
\text { A } \\
\text { C-A } \\
\text { C-A } \\
\text { C-A } \\
\text { C } \\
\text { C } \\
\text { A }\end{array}$ & $\begin{array}{l}\text { M-G } \\
M \\
M \\
M \\
M \\
M \\
\text { M-M } \\
\text { P-M } \\
\text { P-M } \\
M \\
\end{array}$ & $\begin{array}{c}\text { Late } \\
\text { Cam- } \\
\text { panian }\end{array}$ & $\begin{array}{c}\text { Tetra- } \\
\text { lithus } \\
\text { gothicus }\end{array}$ & & $\begin{array}{c}\mathrm{F} \\
\mathrm{R} \\
\mathrm{C} \\
\mathrm{R} \\
\mathrm{F} \\
\mathrm{F} \\
\mathrm{F} \\
\mathrm{F} \\
\mathrm{VR}\end{array}$ & & & VR & & & $\begin{array}{l}\text { A } \\
\text { A } \\
\text { A } \\
\text { A } \\
\text { A } \\
\text { A } \\
\text { A } \\
\text { A } \\
\text { C } \\
\text { A }\end{array}$ & VR & $\begin{array}{l}\mathrm{F} \\
\mathrm{C} \\
\mathrm{F} \\
\mathrm{R} \\
\mathrm{VR} \\
\mathrm{C} \\
\mathrm{R} \\
\mathrm{VR} \\
\mathrm{VR} \\
\mathrm{R} \\
\end{array}$ \\
\hline $\begin{array}{l}16-1,55-56 \\
16-2,55-56 \\
16-3,55-56 \\
\end{array}$ & $\begin{array}{l}132.05 \\
133.55 \\
135.05\end{array}$ & $\underset{\mathrm{A}}{\mathrm{A}}$ & $\begin{array}{l}\mathrm{P}-\mathrm{M} \\
\mathrm{M} \\
\mathrm{M}\end{array}$ & $\begin{array}{l}\text { M. to E. } \\
\text { Campan. }\end{array}$ & $\begin{array}{c}\text { Broinson. } \\
\text { parca }\end{array}$ & & & & & F & & & $\begin{array}{l}\text { A } \\
\text { A } \\
\text { A }\end{array}$ & & $\begin{array}{l}\mathrm{F} \\
\mathrm{C}\end{array}$ \\
\hline $\begin{array}{l}16, \mathrm{CC} \\
17-1,54-55 \\
17, \mathrm{CC} \\
18-1,9-10 \\
19, \mathrm{CC} \\
20, \mathrm{CC} \\
21, \mathrm{CC} \\
22, \mathrm{CC} \\
23, \mathrm{CC} \\
\end{array}$ & $\begin{array}{l}141.00 \\
141.54 \\
150.50 \\
150.59 \\
169.50 \\
179.00 \\
188.50 \\
198.00 \\
207.50\end{array}$ & $\begin{array}{c}\text { C-A } \\
\text { A } \\
\text { A } \\
\text { C-A } \\
\text { C-A } \\
\text { F } \\
\text { F } \\
\text { F } \\
\text { F } \\
\end{array}$ & $\begin{array}{c}\mathrm{P}-\mathrm{M} \\
\mathrm{M} \\
\mathrm{M} \\
\mathrm{P}-\mathrm{M} \\
\mathrm{P} \\
\mathrm{P} \\
\mathrm{P} \\
\mathrm{P} \\
\mathrm{P}\end{array}$ & $\begin{array}{c}\text { Santonian } \\
\text { to } \\
\text { Middle } \\
\text { Turonian }\end{array}$ & (unzoned) & & & & & $\begin{array}{l}\text { VR } \\
\text { F/X }\end{array}$ & & & $\begin{array}{l}\text { A } \\
\text { A } \\
\text { A } \\
\text { A } \\
\text { A } \\
\text { A } \\
\text { A } \\
\text { C } \\
\text { A }\end{array}$ & $\begin{array}{c}\text { VR } \\
\text { R } \\
\text { F } \\
\text { VR }\end{array}$ & $\begin{array}{c}\mathrm{F} \\
\mathrm{C} \\
\mathrm{F} \\
\mathrm{F} \\
\mathrm{F} \\
\mathrm{F} \\
\mathrm{VR}\end{array}$ \\
\hline $28, \mathrm{CC}$ & 255.00 & \multicolumn{2}{|c|}{ (barren) } & $?$ & $?$ & & & & & & & & & & \\
\hline $\begin{array}{l}29-1,34-36 \\
29-2,34-35 \\
29, \text { CC } \\
30-1,24-25 \\
31-1, \text { bottom } \\
34, C C \\
35-1,53-57 \\
35, \text { CC }\end{array}$ & $\begin{array}{l}255.34 \\
256.84 \\
264.50 \\
264.74 \\
283.50 \\
302.50 \\
303.03 \\
312.00\end{array}$ & $\begin{array}{l}\text { C-A } \\
\text { C-A } \\
\text { C } \\
\text { C } \\
\text { A } \\
\text { C } \\
\text { F-C } \\
\text { F-C }\end{array}$ & $\begin{array}{c}M \\
M-G \\
P-M \\
M \\
M \\
P \\
P \\
P\end{array}$ & $\begin{array}{c}\text { Late } \\
\text { Albian }\end{array}$ & $\begin{array}{l}\text { Eiffel- } \\
\text { lithus } \\
\text { turris- } \\
\text { eiffeli }\end{array}$ & C & & $\begin{array}{l}R \\
R\end{array}$ & $\begin{array}{l}\text { C } \\
\text { F } \\
\text { C } \\
\text { C } \\
\text { C } \\
\text { C } \\
\text { VR } \\
\text { VR }\end{array}$ & VR & $\begin{array}{c}\mathrm{VR} \\
\mathrm{R} \\
\mathrm{F} \\
\mathrm{F} \\
\mathrm{VR} / \mathrm{X} \\
\mathrm{F}\end{array}$ & $\begin{array}{l}\mathrm{F} \\
\mathrm{F} \\
\mathrm{F} \\
\mathrm{C} \\
\mathrm{F} \\
\mathrm{C} \\
\mathrm{F} \\
\mathrm{F}\end{array}$ & $\begin{array}{l}\text { A } \\
\text { A } \\
\text { A } \\
\text { A } \\
\text { A } \\
\text { A } \\
\text { A } \\
\text { A }\end{array}$ & $\begin{array}{l}\text { VR } \\
\text { VR }\end{array}$ & $\begin{array}{c}\text { R } \\
\text { R } \\
\text { F } \\
\text { F } \\
\text { C } \\
\text { VR } \\
\text { F } \\
\text { F }\end{array}$ \\
\hline
\end{tabular}

Note: Symbols as in Table 2. 
Table 6. (Continued).

\begin{tabular}{|c|c|c|c|c|c|c|c|c|c|c|c|c|c|c|c|c|}
\hline 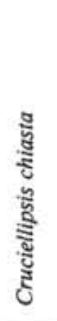 & 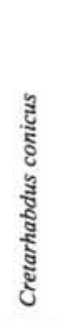 & 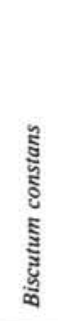 & 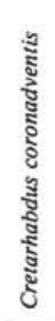 & 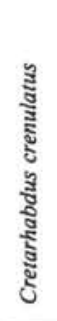 & 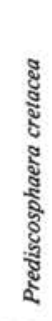 & 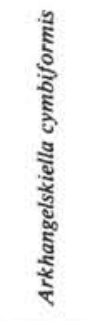 & 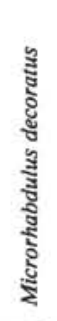 & 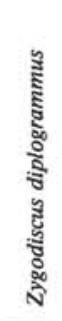 & 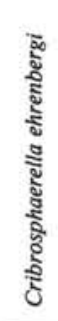 & 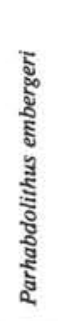 & 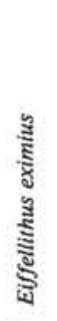 & 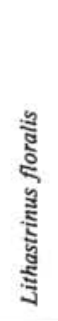 & 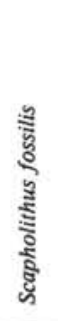 & 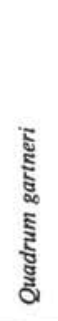 & 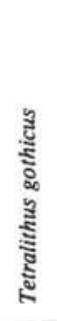 & 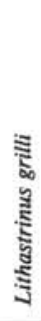 \\
\hline & & C & $F$ & $\begin{array}{l}\text { C } \\
\text { A }\end{array}$ & $\begin{array}{l}\mathrm{A} \\
\mathrm{A}\end{array}$ & $\begin{array}{l}\mathrm{C} \\
\mathrm{C}\end{array}$ & $\begin{array}{l}\mathrm{C} \\
\mathrm{C}\end{array}$ & $\begin{array}{l}\mathrm{C} \\
\mathrm{C}\end{array}$ & $\begin{array}{l}\text { A } \\
\text { A }\end{array}$ & $\begin{array}{c}\text { VR } \\
\text { R }\end{array}$ & & & & & $\begin{array}{l}\mathrm{C} \\
\mathrm{F}\end{array}$ & \\
\hline & VR & $R / X$ & & $\begin{array}{l}\text { C } \\
\text { F } \\
\text { C } \\
\text { C } \\
\text { C } \\
\text { C } \\
\text { C } \\
\text { C } \\
\text { C } \\
\text { C }\end{array}$ & $\begin{array}{l}\text { A } \\
\text { A } \\
\text { A } \\
\text { A } \\
\text { C } \\
\text { A } \\
\text { A } \\
\text { A } \\
\text { A } \\
\text { C }\end{array}$ & $\begin{array}{l}\mathrm{F} \\
\mathrm{C} \\
\mathrm{F} \\
\mathrm{R} \\
\mathrm{F} \\
\mathrm{C} \\
\mathrm{C} \\
\mathrm{F} \\
\mathrm{R} \\
\mathrm{F}\end{array}$ & $\begin{array}{c}\text { C } \\
\text { C } \\
\text { C } \\
C \\
\text { VR } \\
C \\
C \\
C \\
C \\
C \\
\text { R } \\
\end{array}$ & $\begin{array}{c}\text { F } \\
\text { VR } \\
\text { F } \\
\text { F } \\
\text { F } \\
\text { F } \\
\text { C } \\
\text { F } \\
\text { C } \\
\text { R }\end{array}$ & $\begin{array}{l}\mathrm{C} \\
\mathrm{C} \\
\mathrm{C} \\
\mathrm{C} \\
\mathrm{C} \\
\mathrm{C} \\
\mathrm{C} \\
\mathrm{C} \\
\mathrm{F} \\
\mathrm{C}\end{array}$ & $\begin{array}{c}\text { VR } \\
\text { F } \\
\text { F } \\
F \\
\text { C } \\
\text { R } \\
\text { R } \\
\text { F } \\
\text { F } \\
\text { VR }\end{array}$ & $\begin{array}{c}\text { VR } \\
C \\
C \\
\end{array}$ & & & $\begin{array}{c}\mathrm{R} / ? \\
\mathrm{~F} / ? \\
\mathrm{~F} \\
\mathrm{~F} / ? \\
\mathrm{C} \\
\mathrm{R}\end{array}$ & $\begin{array}{c}\text { C } \\
C \\
F \\
\text { C } \\
C \\
\text { F } \\
C \\
F \\
\\
\text { VR }\end{array}$ & \\
\hline & & & & $\begin{array}{l}\text { C } \\
\text { C } \\
\text { A }\end{array}$ & $\begin{array}{l}\mathrm{A} \\
\mathrm{A} \\
\mathrm{A}\end{array}$ & $\begin{array}{c}\mathrm{C} \\
\text { VR } \\
\text { VR }\end{array}$ & $\begin{array}{l}\mathrm{C} \\
\mathrm{F}\end{array}$ & $\begin{array}{l}\mathrm{C} \\
\mathrm{F} \\
\mathrm{C}\end{array}$ & $\begin{array}{l}\mathrm{C} \\
\mathrm{C} \\
\mathrm{F}\end{array}$ & $\begin{array}{l}\mathrm{C} \\
\mathrm{F} \\
\mathrm{F}\end{array}$ & $\begin{array}{l}\mathrm{F} \\
\mathrm{C} \\
\mathrm{C}\end{array}$ & & & & & \\
\hline & & & & $\begin{array}{l}\text { C } \\
\text { A } \\
\text { C } \\
\text { C } \\
\text { A } \\
\text { F } \\
\text { F } \\
\text { F } \\
\text { F }\end{array}$ & $\begin{array}{l}\text { A } \\
\text { A } \\
\text { A } \\
\text { C } \\
\text { A } \\
\text { F } \\
\text { C } \\
\text { F } \\
\text { F }\end{array}$ & $\mathrm{VR} / \mathrm{X}$ & $\begin{array}{c}\text { VR } \\
\text { C } \\
\text { C } \\
\text { F } \\
\text { VR } \\
\\
\text { VR } \\
\text { VR }\end{array}$ & $\begin{array}{c}\mathrm{C} \\
\mathrm{F} \\
\mathrm{F} \\
\mathrm{C} \\
\mathrm{R} / \mathrm{X}\end{array}$ & $\begin{array}{c}\mathrm{C} \\
\mathrm{F} \\
\mathrm{C} \\
\mathrm{F} \\
\mathrm{VR}\end{array}$ & $\begin{array}{c}\text { VR } \\
\text { VR } \\
C \\
\text { VR } \\
C \\
\text { VR } \\
\text { VR }\end{array}$ & $\begin{array}{c}\mathrm{C} \\
\mathrm{A} \\
\mathrm{A} \\
\mathrm{F} \\
\mathrm{C} \\
\text { VR } \\
\text { VR } \\
\text { VR } \\
\text { VR } \\
\end{array}$ & & & & & $\begin{array}{l}F \\
F\end{array}$ \\
\hline F & & $\begin{array}{l}\text { F } \\
\text { C } \\
\text { A } \\
\text { A } \\
\text { A } \\
\text { A } \\
\text { A } \\
\text { A }\end{array}$ & VR & $\begin{array}{c}\text { VR } \\
\text { R } \\
\text { F } \\
\text { C } \\
\text { F } \\
\text { C } \\
\text { F }\end{array}$ & $\begin{array}{l}\mathrm{C} \\
\mathrm{C} \\
\mathrm{C} \\
\mathrm{F}\end{array}$ & & & $\begin{array}{l}\text { C } \\
\text { C } \\
\text { C } \\
\text { C } \\
\text { C } \\
\text { C } \\
\text { C } \\
\text { F }\end{array}$ & F & $\begin{array}{l}\text { C } \\
\text { C } \\
\text { F } \\
\text { C } \\
\text { C } \\
\text { C } \\
C\end{array}$ & & $\begin{array}{c}\mathrm{R} \\
\mathrm{VR}\end{array}$ & VR & & & \\
\hline
\end{tabular}


P. ČEPEK

Table 6. (Continued).

\begin{tabular}{|c|c|c|c|c|c|c|c|c|c|c|c|c|c|c|c|}
\hline $\begin{array}{c}\text { Sample } \\
\text { (interval } \\
\text { in } \mathrm{cm} \text { ) }\end{array}$ & $\begin{array}{l}\text { Sub- } \\
\text { bottom } \\
\text { Depth } \\
\text { (m) }\end{array}$ & & & & & 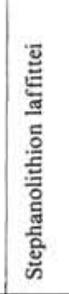 & 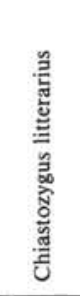 & 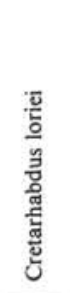 & 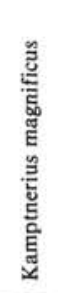 & 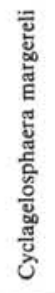 & 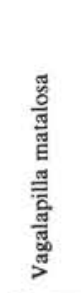 & 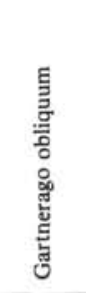 & 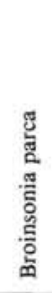 & 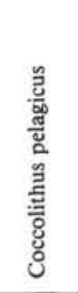 & 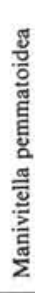 \\
\hline $\begin{array}{c}466-11-1,45-46 \\
11, C C \\
\end{array}$ & $\begin{array}{l}88.45 \\
93.50 \\
\end{array}$ & $\begin{array}{l}\mathrm{A} \\
\mathrm{A}\end{array}$ & $\begin{array}{l}\mathrm{M} \\
\mathrm{M}\end{array}$ & $\begin{array}{c}\text { E. Maa. } \\
\text { to L. Cam. }\end{array}$ & $\begin{array}{l}\text { Tetral. } \\
\text { trifidus }\end{array}$ & $\begin{array}{l}\mathrm{R} \\
\mathrm{F}\end{array}$ & $\begin{array}{l}\mathrm{C} \\
\mathrm{C}\end{array}$ & $\begin{array}{l}\mathrm{C} \\
\mathrm{F} \\
\end{array}$ & VR & & & & $\begin{array}{c}\mathrm{F} \\
\text { VR }\end{array}$ & $R(+)$ & $\begin{array}{l}\mathrm{F} \\
\mathrm{C}\end{array}$ \\
\hline $\begin{array}{l}12-1,35-36 \\
12, \mathrm{CC} \\
13-1,40-41 \\
13-3,35-37 \\
13-5,84-85 \\
13, \mathrm{CC} \\
14, \mathrm{CC} \\
15-1,42-43 \\
15-4,42-43 \\
15, \mathrm{CC} \\
\end{array}$ & $\begin{array}{r}93.85 \\
103.00 \\
103.40 \\
106.35 \\
109.84 \\
112.50 \\
122.00 \\
122.42 \\
126.92 \\
131.50 \\
\end{array}$ & $\begin{array}{l}\text { A } \\
\text { A } \\
\text { A } \\
\text { A } \\
\text { C-A } \\
\text { C-A } \\
\text { C-A } \\
\text { C } \\
\text { C } \\
\text { A }\end{array}$ & $\begin{array}{c}\mathrm{M}-\mathrm{G} \\
\mathrm{M} \\
\mathrm{M} \\
\mathrm{M} \\
\mathrm{M} \\
\mathrm{M} \\
\mathrm{P}-\mathrm{M} \\
\mathrm{P}-\mathrm{M} \\
\mathrm{P}-\mathrm{M} \\
\mathrm{M}\end{array}$ & $\begin{array}{l}\text { Late } \\
\text { Cam- } \\
\text { panian }\end{array}$ & $\begin{array}{c}\text { Tetra- } \\
\text { lithus } \\
\text { gothicus }\end{array}$ & $\mathbf{R}$ & $\begin{array}{l}\text { C } \\
\text { C } \\
\text { C } \\
\text { F } \\
\text { C } \\
\text { C } \\
\text { F } \\
\text { F } \\
\text { F } \\
\text { C }\end{array}$ & $\begin{array}{l}\mathrm{C} \\
\mathrm{R} \\
\mathrm{F} \\
\mathrm{F} \\
\mathrm{R} \\
\mathrm{C} \\
\mathrm{R} \\
\mathrm{R} \\
\mathrm{R} \\
\mathrm{VR}\end{array}$ & & & & $\begin{array}{c}\text { VR } \\
\text { R } \\
\text { VR }\end{array}$ & $\begin{array}{c}\text { VR } \\
F \\
F \\
F \\
C \\
F \\
F \\
\text { FR } \\
\text { F } \\
\text { VR }\end{array}$ & & $\begin{array}{l}\text { F } \\
\text { C } \\
\text { C } \\
\text { C } \\
\text { C } \\
\text { C } \\
\text { F } \\
\text { F } \\
\text { C } \\
\text { F }\end{array}$ \\
\hline $\begin{array}{l}16-1,55-56 \\
16-2,55-56 \\
16-3,55-56\end{array}$ & $\begin{array}{l}132.05 \\
133.55 \\
135.05\end{array}$ & $\begin{array}{c}\mathrm{A} \\
\mathrm{C}-\mathrm{A} \\
\mathrm{A}\end{array}$ & $\begin{array}{c}\mathrm{P}-\mathrm{M} \\
\mathrm{M} \\
\mathrm{M} \\
\end{array}$ & $\begin{array}{l}\text { M. to E. } \\
\text { Campan. }\end{array}$ & $\begin{array}{c}\text { Broinson. } \\
\text { parca }\end{array}$ & $F / X$ & $\begin{array}{l}\mathrm{F} \\
\mathrm{F} \\
\mathrm{C}\end{array}$ & VR & & $\mathrm{F}$ & & & $\begin{array}{l}\text { VR } \\
\mathrm{F} \\
\mathrm{R} \\
\end{array}$ & & $\begin{array}{l}\mathrm{C} \\
\mathrm{C} \\
\mathrm{C}\end{array}$ \\
\hline $\begin{array}{l}16, \mathrm{CC} \\
17-1,54-55 \\
17, \mathrm{CC} \\
18-1,9-10 \\
19, \mathrm{CC} \\
20, \mathrm{CC} \\
21, \mathrm{CC} \\
22, \mathrm{CC} \\
23, \mathrm{CC}\end{array}$ & \begin{tabular}{l|}
141.00 \\
141.54 \\
150.50 \\
150.59 \\
169.50 \\
179.00 \\
188.50 \\
198.00 \\
207.50 \\
\end{tabular} & $\begin{array}{c}\text { C-A } \\
\text { A } \\
\text { A } \\
\text { C-A } \\
\text { C-A } \\
\text { F } \\
\text { F } \\
\text { F } \\
\text { F }\end{array}$ & $\begin{array}{c}\mathrm{P}-\mathrm{M} \\
\mathrm{M} \\
\mathrm{M} \\
\mathrm{P}-\mathrm{M} \\
\mathrm{P} \\
\mathrm{P} \\
\mathrm{P} \\
\mathrm{P} \\
\mathrm{P}\end{array}$ & $\begin{array}{c}\text { Santonian } \\
\text { to } \\
\text { Middle } \\
\text { Turonian }\end{array}$ & (unzoned) & & $\begin{array}{c}C \\
C \\
C \\
\text { VR } \\
\text { F } \\
\text { VR } \\
V R / X\end{array}$ & & & & & $\begin{array}{c}\mathrm{VR} \\
\mathrm{VR} / \mathrm{X}\end{array}$ & & & $\begin{array}{l}\text { C } \\
\text { C } \\
\text { C } \\
\text { C } \\
\text { C } \\
\text { F } \\
\text { F } \\
\text { C } \\
\text { F }\end{array}$ \\
\hline $28, \mathrm{CC}$ & 255.00 & \multicolumn{2}{|c|}{ (barren) } & $?$ & $?$ & & & & & & & & & & \\
\hline $\begin{array}{l}29-1,34-36 \\
29-2,34-35 \\
29, C C \\
30-1,24-25 \\
31-1, \text { bottom } \\
34, C C \\
35-1,53-57 \\
35, C C\end{array}$ & $\begin{array}{l}255.34 \\
256.84 \\
264.50 \\
264.74 \\
283.50 \\
302.50 \\
303.03 \\
312.00\end{array}$ & $\begin{array}{l}\text { C-A } \\
\text { C-A } \\
\text { C } \\
\text { C } \\
\text { A } \\
\text { C } \\
\text { F-C } \\
\text { F-C }\end{array}$ & $\begin{array}{c}\mathrm{M} \\
\mathrm{M}-\mathrm{G} \\
\mathrm{P}-\mathrm{M} \\
\mathrm{M} \\
\mathrm{M} \\
\mathrm{P} \\
\mathrm{P} \\
\mathrm{P}\end{array}$ & $\begin{array}{c}\text { Late } \\
\text { Albian }\end{array}$ & $\begin{array}{l}\text { Eiffel- } \\
\text { lithus } \\
\text { turris- } \\
\text { eiffeli }\end{array}$ & $\begin{array}{l}\text { F } \\
\text { VR } \\
F\end{array}$ & $\begin{array}{l}\mathrm{F} \\
\mathrm{C} \\
\mathrm{F} \\
\mathrm{C} \\
\mathrm{C} \\
\mathrm{C} \\
\mathrm{C} \\
\mathrm{R}\end{array}$ & $\mathrm{C} / \mathrm{X}$ & & VR & $\begin{array}{c}\mathrm{VR} / \mathrm{X} \\
\mathrm{C}\end{array}$ & & & & $\begin{array}{l}\mathrm{C} \\
\mathrm{C} \\
\mathrm{C} \\
\mathrm{C} \\
\mathrm{C} \\
\mathrm{C} \\
\mathrm{F} \\
\mathrm{F}\end{array}$ \\
\hline
\end{tabular}


Table 6. (Continued).

\begin{tabular}{|c|c|c|c|c|c|c|c|c|c|c|c|c|c|c|c|}
\hline 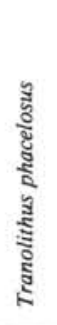 & 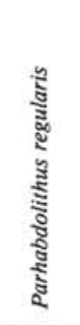 & 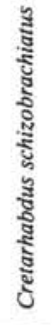 & 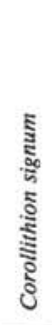 & 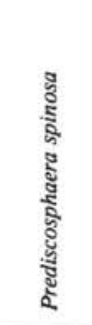 & 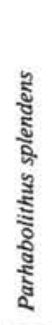 & 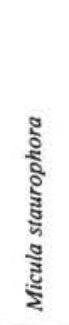 & 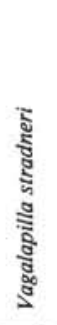 & 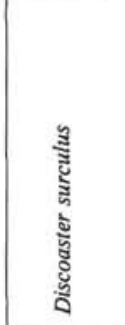 & 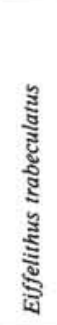 & 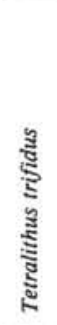 & 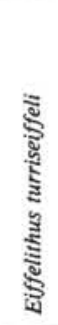 & 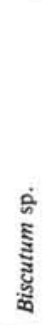 & 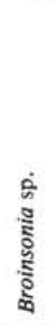 & 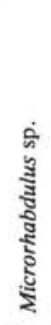 & $\begin{array}{l}\text { के } \\
\text { ș } \\
\text { ș } \\
\text { है }\end{array}$ \\
\hline F & $\mathrm{VR} / \mathrm{X}$ & $\begin{array}{c}R \\
\text { VR }\end{array}$ & & & & $\begin{array}{c}\text { VR } \\
\text { R }\end{array}$ & F & & & $\underset{\text { VR }}{\mathrm{F}}$ & $\begin{array}{l}\mathrm{C} \\
\mathrm{C}\end{array}$ & & & & $\begin{array}{l}\mathrm{C} \\
\mathrm{R}\end{array}$ \\
\hline $\begin{array}{c}\text { VR } \\
\text { F } \\
\text { R } \\
\text { VR } \\
\text { VR } \\
\text { VR } \\
\text { R } \\
\text { C } \\
\text { R }\end{array}$ & $\begin{array}{c}\mathrm{R} \\
\mathrm{VR}\end{array}$ & & & & & $\begin{array}{l}\mathrm{F} \\
\mathrm{F} \\
\mathrm{F} \\
\mathrm{F} \\
\mathrm{C} \\
\mathrm{C} \\
\mathrm{F} \\
\mathrm{F} \\
\mathrm{F} \\
\mathrm{C} \\
\end{array}$ & VR & $\mathrm{VR} / \mathrm{X} /(+)$ & F & & $\begin{array}{l}\mathrm{C} \\
\mathrm{C} \\
\mathrm{C} \\
\mathrm{F} \\
\mathrm{C} \\
\mathrm{C} \\
\mathrm{C} \\
\mathrm{F} \\
\mathrm{F} \\
\mathrm{C} \\
\end{array}$ & & & $\begin{array}{c}\mathrm{C} \\
\mathrm{VR}\end{array}$ & \\
\hline $\begin{array}{l}R \\
R \\
C\end{array}$ & $\mathrm{~F}$ & & & & & $\begin{array}{l}\mathrm{C} \\
\mathrm{F} \\
\mathrm{C} \\
\end{array}$ & VR & & & & $\begin{array}{l}\mathrm{C} \\
\mathrm{F} \\
\mathrm{C}\end{array}$ & & & & \\
\hline $\begin{array}{c}\mathrm{F} \\
\mathrm{F} \\
\text { F/X } \\
\text { VR }\end{array}$ & $\begin{array}{l}\mathrm{C} / \mathrm{X} \\
\mathrm{F} / \mathrm{X}\end{array}$ & & & & & $\begin{array}{c}\text { C } \\
\text { C } \\
\text { F } \\
\text { F/X } \\
\text { VR } \\
\text { F } \\
\text { VR } \\
\text { R } \\
\text { F } \\
\end{array}$ & & & $\begin{array}{l}\text { VR } \\
\text { VR }\end{array}$ & & $\begin{array}{c}\text { C } \\
\text { C } \\
\text { F } \\
\text { VR } \\
\text { F } \\
\text { VR } \\
\text { VR }\end{array}$ & & & & \\
\hline $\begin{array}{c}\mathrm{F} \\
\mathrm{F} \\
\mathrm{C} \\
\mathrm{F} \\
\mathrm{C} \\
\mathrm{VR} \\
\mathrm{F}\end{array}$ & & & VR & $\mathrm{VR} /(\mathrm{X})$ & C & & $\begin{array}{l}\text { C } \\
\text { F } \\
\text { F } \\
\text { C } \\
\text { F } \\
\text { F } \\
\text { F } \\
\text { F }\end{array}$ & & & & $\begin{array}{l}\text { C } \\
\text { E } \\
\text { A } \\
\text { A } \\
\text { A } \\
\text { C } \\
\text { C } \\
\text { C }\end{array}$ & $R$ & F & & \\
\hline
\end{tabular}


P. ČEPEK

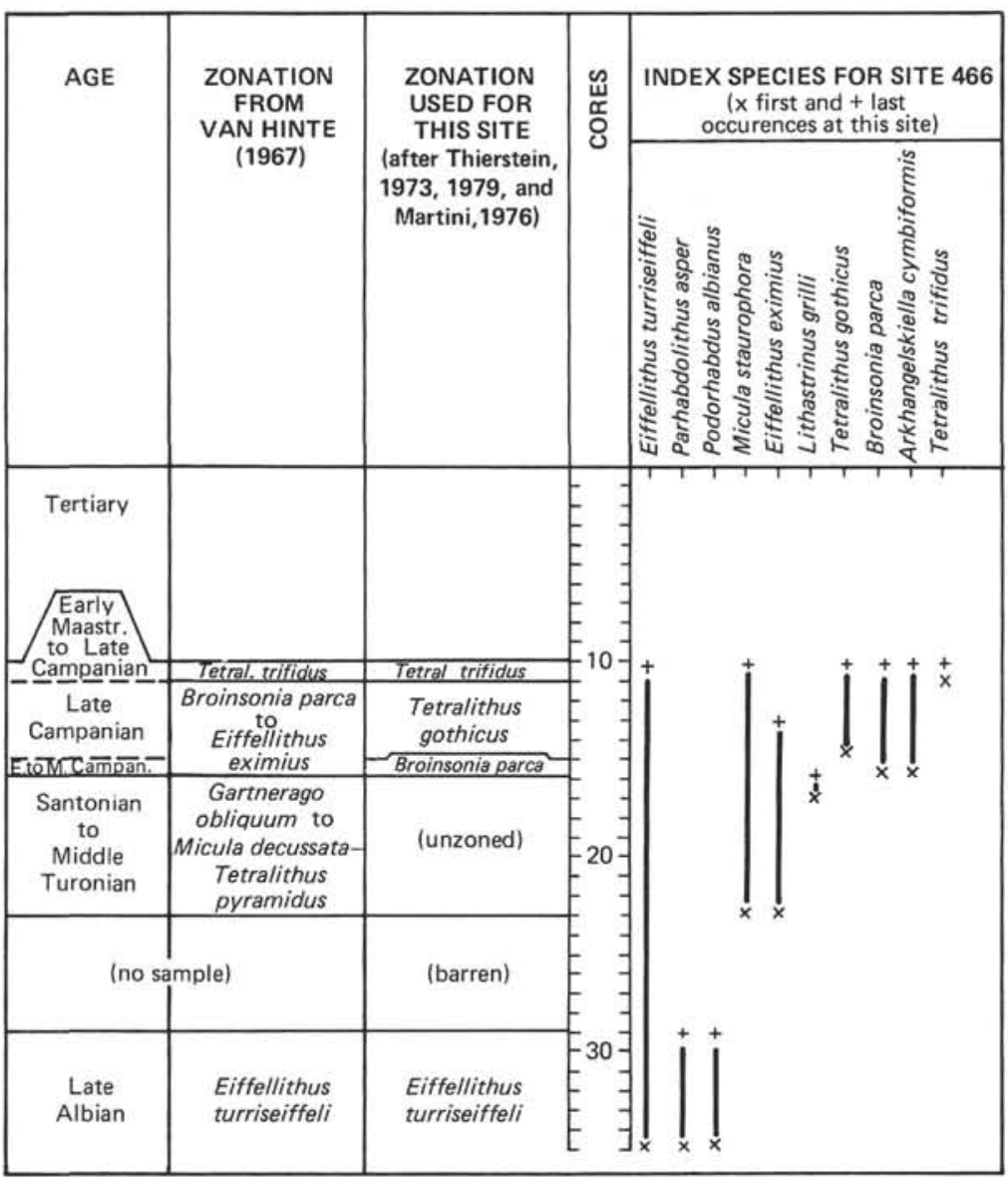

Figure 7. Ranges of index species at Site 466 and correlation of the zonation used with that according to van Hinte (1976). 